\title{
Influence of Gravity on the Stability of Evaporative Convection Regimes
}

\author{
V. B. Bekezhanova ${ }^{1,2}$. I. A. Shefer ${ }^{2}$
}

Received: date / Accepted: date

\begin{abstract}
The characteristics of convective regimes in 27 a two-layer system have been investigated in the framework of the Boussinesq approximation of the Navier- 28 Stokes equations. An exact invariant solution of the ${ }_{29}$ convection equations is used to describe a joint sta- 30 tionary flow of an evaporating liquid and a gas-vapor ${ }_{31}$ mixture in a horizontal channel. Thermodiffusion ef- 32 fects in the gas-vapor phase are additionally taken into 33 account in the governing equations and interface con- 34 ditions. The influence of gravity and thickness of the 35 liquid layer on the hydrodynamical, thermal and con- 36 centration characteristics of the regimes has been in- 37 vestigated. Flows of the pure thermocapillary, mixed 38 and Poiseuille's types are specified for different values ${ }_{39}$ of the problem parameters. The linear stability of the 40 evaporative convection regimes has been studied. The 41 types and properties of the arising perturbations have 42 been investigated and the critical characteristics of the ${ }_{43}$ stability have been obtained. Disturbances can lead to 44 the formation of deformed convective cells, vortex and ${ }_{45}$ thermocapillary structures. The change of the instabil- 46 ity types and threshold thermal loads occurs with the ${ }_{47}$ increasing thickness of the liquid layer and gravity ac- 48 tion.
\end{abstract}

Keywords Evaporative convection - Exact solution · Characteristic perturbations · Stability

This work was supported by the Russian Foundation for Basic 54 Research (project No. 17-08-00291).

1 Department of Differential Equations of Mechanics, Insti- 56 tute of Computational Modelling SB RAS, 660036, Akadem- ${ }_{57}$ gorodok, 50/44, Krasnoyarsk, Russia

E-mail: vbek@icm.krasn.ru .

2 Institute of Mathematics and Computer Science, Siberian 59 Federal University, 660041, Svobodny, 79, Krasnoyarsk, Rus- 60 sia

\section{Introduction}

Convective flows with evaporation/condensation in different systems have been the subject of a detailed investigation in the past few decades (for a review, see Berg et al. 1966, Hoke and Chen 1992, Molenkamp 1998, Kabov et al. 2015). The traditional application fields for the flows of evaporating liquids are chemical engineering and materials science (Mancini and Maza 2004, Nie and Kumacheva 2008, Scheid et al. 2012) and thermophysics (Bar-Cohen and Wang 2012, Kandlikar et al. 2013, Kabov et al. 2015). The interest to the study of heat and mass transfer processes is due to the rapid development of biotechnologies and chemical industry, and tremendous advances in mini- and microscale cooling technologies and thermostabilization methods in high-performance electronic systems (such as micro heat exchanger in power packages, life-support setups of orbital platforms, etc.) as well as due to the preparation of experiments on the International Space Station in the framework of the scientific project "Convection and Interfacial Mass Exchange" (CIMEX) of the European Space Agency.

A thorough analysis of the influence of different factors on the flow structure is necessary to improve the existing fluidic technologies or to develop another approach, radically different from the conventional practice using evaporating liquids and gas-vapor mixtures as working fluids. The investigation of the characteristics and features of evaporative convection was performed both experimentally (Colinet et al. 2003, Mancini and Maza 2004, Iorio et al. 2007. Reutov et al. 2007. Kimball et al. 2012, Lyulin and Kabov 2014, Shi et al. 2017) and theoretically in the framework of different approaches. At present, there is no general universal mathematical theory to describe the dynamics of the two-layer system 
with phase transition. Most of the theoretical and nu- 54 merical investigations are performed in the framework 55 of the mathematical models based on the fundamen- 56 tal laws of the classical continuum mechanics and ther- 57 modynamics. One of the most generally employed ap- 58 proaches to describe evaporative convection is founded 59 on using the Navier-Stokes equations and their ap- 60 proximations, in particular, the Oberbeck-Boussinesq ${ }_{61}$ one. Upon that, an additional difficulty in the problem 62 is the formulation of the boundary conditions taking 63 into account the evaporation/condensation at the inter- 64 face in order to correctly close the evaporative convec- 65 tion problem. The conditions are derived on the basis 66 of some hypotheses with respect to the interface and 67 the occurring physical processes, which guarantee the 68 fulfillment of the conservation laws (Prosperetti 1979, 69 Margerit et al. 2003, Das and Ward 2007, Frezzotti 70 2011, Kuznetsov 2011, Goncharova 2012, Goncharova 71 et al. 2013). With the help of the long-wave approxi- 72 mation of the basic system of equations the convective 73 flows accompanied by the mass transfer through the 74 interface were studied analytically and numerically in 75 (Oron et al. 1997, Shklyaev and Fried 2007, Kuznetsov 76 and Andreev 2013, Kabova et al. 2014, Goncharova 77 and Rezanova 2015). Analogous problems in the com- 78 plete statement were considered in (Iorio et al. 2009, 79 Kuznetsov 2011, Goncharova 2012, Goncharova et al. 80 2013, Bekezhanova and Goncharova 2016). Numerical ${ }_{81}$ simulation of two-phase dynamics of thermocapillary 82 flows and of phase transition processes in a channel was 83 realized on basis the Navier-Stokes and energy equa- 84 tions in (Saenz et al. 2013, 2014, Li et al. 2018) .

One way to examine in detail the influence of dif- 86 ferent thermal, mechanical and physical-chemical fac- 87 tors on the character and intensity of two-layer flows is 88 modeling the heat and mass transfer processes on the 89 basis of exact solutions of the convection equations. It 90 is a very useful tool allowing one to qualitatively spec- 91 ify the main physical mechanisms defining the struc- 92 ture of the basic flow and to investigate the degrees 93 and nature of the influence of particular physical fac- 94 tors and their mutual combinations. The Navier-Stokes 95 and Oberbeck - Boussinesq equations possess rich group 96 properties, in as much as they were formulated based on 97 the postulates, which imply the natural symmetry prop- 98 erties of space-time and of a fluid moving in the space 99 (Pukhnachev 2006). The group properties allow one toroo construct exact solutions of the equations. These par-101 ticular solutions being of the group origin, are of partic-102 ular value, since they conserve the symmetry propertiesio3 provided by the derivation of the basic equations. Thero4 group nature of the solution ensures its physical plau-105 sibility and realizability (Andreev et al. 1998, 2003). ${ }_{106}$
The flows with evaporation/condensation are characterized by the presence of the temperature gradient, which arises due to the decrease of the average kinetic energy of a liquid volume. For the first time the exact solution describing the convective flows being under the action of the arbitrary oriented temperature gradient was obtained in (Ostroumov 1952). An analogous solution of the Oberbeck - Boussinesq equations for the flow in a horizontal layer with the applied longitudinal temperature gradient was again derived in (Birikh 1966). Later, the Ostroumov-Birikh solution was generalized for describing convection in a plane two-layer system with the mass transfer through the interface for the cases "liquid-liquid" (Shliomis and Yakushin 1972) and "liquid-vapor-gas mixture" (Goncharova and Rezanova 2014). In the latter work, vapor was supposed to be a passive admixture, and vapor diffusion in the gas was described by the diffusion equation, and additionally, the thermocapillarity of the interface was taken into account. A threedimensional analogue of the Ostroumov-Birikh solution for the evaporative convection problem was constructed in (Goncharova and Kabov 2016). These generalizations of the Ostroumov - Birikh solution additionally admit considering the thermal diffusion effects (the Soret and Dufour effects) in the gas phase. The group nature of the Ostroumov-Birikh type solutions was proved in (Pukhnachev 2000). The examples of other exact solutions describing the dynamics of evaporating liquids can be found in (Kuznetsov 2011, Kuznetsov and Andreev 2013).

The Ostroumov - Birikh type solutions allow one to analyze the influence of various factors on the characteristics of the evaporative convection regimes, including the stability properties, as well as to evaluate the character and degree of the influence of the boundary condition type for the temperature and vapor concentration functions. The structure of joint flows of the evaporating liquid and gas-vapor mixture depending on the gravity intensity, values of the gas flow rate and applied longitudinal temperature gradient on the channel walls was investigated (Goncharova et al. 2013, Goncharova and Rezanova 2014). The influence of the thermal diffusion effects on the evaporation intensity was studied and the theoretical results were compared with the experimental data (Goncharova et al. 2015). The analysis and classification of the two-layer flows, which can be described by the Ostroumov-Birikh solution analogues, depending on the boundary condition type for the vapor concentration function, flow topology, structure of the temperature field and inclusion/exception of the Soret effect, were presented in (Bekezhanova and Goncharova 2016). 
Evaporation cools the liquid surface, it leads to the ${ }_{54}$ formation of potentially unstable fluid stratification. 55 Thereby, the surface tension changes as well. It can re- 56 sult in the appearance of instabilities of different na- 57 ture. The analysis of possible mechanisms of instability 58 and finding the conditions and effective ranges of the 59 problem parameters ensuring the stability of the basic 60 state of the two-layer system, are necessary both be- 61 fore realizing experiments and during the preliminary 62 stages of the development of special equipment using ${ }_{63}$ evaporating liquids as a working medium. The main 64 part of the investigations at this point concerns the 65 stability problems for thin liquid film flows (Klentz- 66 man and Ajaev 2009, Liu and Kabov 2012) or equi- 67 librium state (Burelbach et al. 1988, Oron 2000, Col- 68 inet et al. 2001, Margerit et al. 2003, Merkt and Beste- 69 horn 2003, Ozen and Narayanan 2004, Haut and Col- 70 inet 2005, Sultan et al. 2005, Shklyaev and Fried 2007, 71 Narendranath et al. 2014). The stability of joint flows 72 of a volatile liquid and co-current gas flux in a horizon- 73 tal channel described by the Ostroumov-Birikh type 74 solutions, was investigated in (Bekezhanova and Gon- 75 charova 2016, Rodionova and Rezanova 2016, Rezanova 76 and Shefer 2016, Bekezhanova et al. 2017). In (Rodi- 77 onova and Rezanova 2016) the linearized equations for the amplitudes of the normal disturbances of the basic solution, long-wave asymptotics of the eigenvalues and eigenfunctions were obtained and the stability with regard to long-wave normal perturbations was proved. 78 The spectrum of the characteristic perturbations of the velocity, temperature and vapor concentration was cal- 79 culated (Rezanova and Shefer 2016, Bekezhanova et al.

2017). The dependence of the type and structure of ${ }_{80}$ the perturbations on the system geometry, disturbance ${ }_{81}$ wave-length and intensity of external actions (the tem- ${ }_{82}$ perature gradient on the channel walls and flow rate of ${ }_{83}$ the working media) was studied for the case of the equal ${ }_{84}$ thermal load applied on the boundaries of the flow do- ${ }_{85}$ main. It was found that the perturbations could lead to ${ }_{86}$ the formation of a vortex, thermocapillary and hybrid ${ }_{87}$ structures corresponding to different mechanisms of the ${ }_{88}$ instability. In the case of different thermal loads on the channel walls the influence of the intensity and charac- ${ }_{90}$ ter of the thermal load (heating/cooling), gas flow rate ${ }_{91}$ and amplitude of the initial perturbations on the type ${ }_{92}$ of the arising instabilities was studied in (Bekezhanova and Goncharova 2016). The stability of the basic flow is ${ }^{93}$ ensured only under quite small thermal gradients and ${ }^{94}$ gas flow rates. The instability can appear due to the ${ }^{95}$ generation of monotonic and oscillatory regimes. The ${ }^{96}$ first one is characterized by the formation of the vor- ${ }^{97}$ tex and thermocapillary structures. In the other regime ${ }_{99}^{98}$ "pulsatory" vortices can arise.
The character and structure of the two-layer flow and evaporation/condensation effects are defined by the combined effect of the following basic factors: (i) temperature regime, (ii) geometry of the system (in particular, thicknesses of the liquid and gas layers), (iii) intensity of the gravity action, (iv) medium flow rates and (v) thermophysical properties of the media. Each of them makes a particular contribution. In the present work we focus on the factors (ii) and (iii) to better understand the character of the gravity effect and to estimate the change of critical characteristics of the stability depending on the gravity action and thickness of the liquid layer. The pattern of the evaporative convection regimes in gravitational fields of various intensity in the systems with different depth of the fluid layer is investigated based on the generalization of the Ostroumov-Birikh solution, taking into account thermal diffusion effects in the gas-vapor layer. The stability of the regimes is studied in the framework of the linear theory. The dependence of the evaporative mass flow rate, critical characteristics of the linear stability and of the typical forms of the arising perturbations on the gravity intensity are studied. The mechanisms leading to changing the flow structure are specified.

\section{Mathematical model}

\subsection{General equations and governing parameters}

Let a volatile liquid and gas-vapor mixture fill the plane infinite horizontal channel with solid impermeable walls (the general scheme is given in Fig. 1). The vertical coordinate $y$ is taken to be directed opposite to the uniform gravity acceleration $\mathbf{g}=(0,-g)$. The thicknesses of the liquid and gas phase are $h_{1}$ and $h_{2}$, respectively. The interface $\Gamma$ is the thermocapillary boundary $y=0$. The tangential forces act along $\Gamma$ and the surface tension $\sigma$ linearly depends on the temperature $\sigma=\sigma_{0}-æ\left(T-T_{0}\right) ; \sigma_{0}, T_{0}$ are the characteristic values of the surface tension and liquid temperature, respectively, $æ>0$ is the temperature coefficient of the surface tension.

We consider small temperature variations across the liquid and gas phases so that the Boussinesq approximation of the Navier-Stokes equations is valid to describe the stationary motion of each medium. Vapor is assumed to be a passive impurity and the vapor diffusion in the gas is described by the diffusion equation. The Dufour and Soret effects (the effects of diffusive thermal conductivity and thermodiffusion) in the gas 


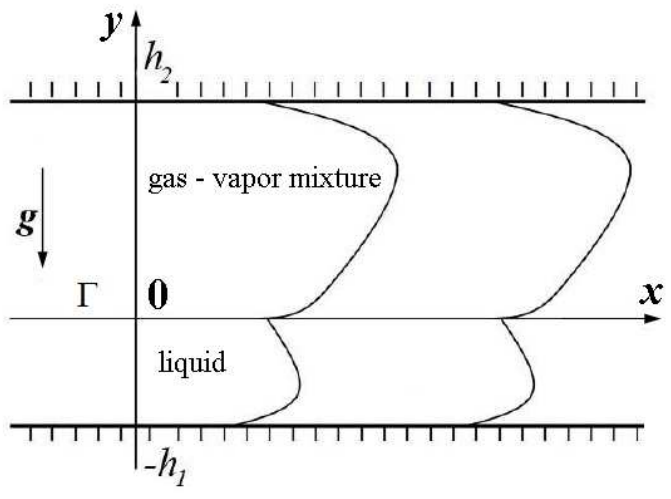

Fig. 1 Configuration of the system

phase are additionally taken into account. Then, the ${ }_{42}^{41}$ governing equations are

$(\mathbf{v} \cdot \nabla) \mathbf{v}=-\frac{1}{\rho} \nabla p^{\prime}+\nu \Delta \mathbf{v}-\mathbf{g}(\beta T+\underline{\gamma C})$,

$\operatorname{div} \mathbf{v}=0$

$\mathbf{v} \cdot \nabla T=\chi(\Delta T+\underline{\delta \Delta C})$,

$\mathbf{v} \cdot \nabla C=D(\Delta C+\alpha \Delta T)$,

where the marked terms and (2.4) are considered to model the flows in the upper layer. The following nota- ${ }^{49}$ tions are introduced: $\mathbf{v}=(u, v)$ is the velocity vector, ${ }^{50}$ $p^{\prime}$ is the modified pressure (the deviation of pressure ${ }^{51}$ $p$ from the hydrostatic one $\left.p^{\prime}=p-\rho \mathbf{g} \cdot \mathbf{x}, \mathbf{x}=(x, y)\right),{ }^{52}$ $T$ is the temperature, $C$ is the vapor concentration, $\rho$ is ${ }^{53}$ the density (the reference value of the density), $\nu$ is ${ }^{54}$ the kinematic viscosity coefficient, $\beta$ is the coefficient ${ }^{55}$ of thermal expansion, $\gamma$ is the concentration density ${ }^{56}$ coefficient, $\chi$ is the coefficient of heat diffusivity, $D$ is ${ }^{57}$ the coefficient of vapor diffusion, the coefficients $\delta$ and ${ }^{58}$ $\alpha$ characterize the Dufour and Soret effects in the gas- ${ }^{59}$ vapor layer, respectively. $\left.u_{1}\right|_{y=-h_{1}}=0,\left.\quad u_{2}\right|_{y=h_{2}}=0$.

$T_{1 \mid y=-h_{1}}=A_{1} x+\vartheta^{-}, \quad T_{2 \mid y=h_{2}}=A_{2} x+\vartheta^{+}$.

For the vapor concentration the condition of zero vapor flux is set

$\left.\left(\frac{\partial C}{\partial y}+\alpha \frac{\partial T}{\partial y}\right)\right|_{y=h_{2}}=0$.

It should be noted that the consideration of the Soret effect in the latter relation is justified in the limited range of values of the problem parameters. The temperature effect can be neglected in (2.8) under certain conditions with an error not exceeding 1-2\%. On the one hand, condition (2.8) is used legitimately at the minor temperature and concentration gradients (Landau and Lifshitz 1987). On the other hand, any considerable temperature deviation in the system and deviation in the vapor concentration in the upper layer (more than $0.5 \%$ ) are observed through these values of the longitudinal temperature gradients $A_{1}$ and $A_{2}$, which can be considered to be moderate ones, which makes a sufficient contribution to the formation of the thermal regime and concentration field. Thus, the question of taking into consideration the Soret effect in the boundary condition (2.8) requires additional analysis in each particular case.

It is assumed that the interface $\Gamma$ remains a nondeformed and flat surface. Then, the equation $y=0$ defines $\Gamma$ when constructing solution (2.5). In the strict sense, the problem at hand becomes a model one in the framework of the assumption about the plane interface. But this assumption allows one to completely take into account the dynamic condition. Considering the physical factors of the interface non-deformability results in another boundary condition statement (Zeytounian 1998, Nepomnyashchy et al. 2002). 
At the interface the continuity of the velocity and temperature is required

$\left.u_{1}\right|_{y=0}=\left.u_{2}\right|_{y=0},\left.\quad T_{1}\right|_{y=0}=\left.T_{2}\right|_{y=0}$.

The relation $a_{1}^{1}=a_{1}^{2}=A$ is valid due to the condition of temperature continuity on $\Gamma$ and the temperature ${ }_{42}$ distribution on the interface takes the form

$T_{j}=\left(A+a_{2}^{j} y\right) x+\vartheta_{j}(y), \quad j=1,2$.

Note that solution (2.5) admits both the case of $a_{46}$ equal thermal load on the channel wall, when $A_{1}={ }_{47}$ $A_{2}=A$ and $\vartheta^{+}=\vartheta^{-}$, and the case $A_{1} \neq A_{2}$, when the ${ }_{48}$ longitudinal temperature gradient $A$ on $\Gamma$ is carried out ${ }_{49}$ over the values of $A_{1}$ and $A_{2}$ (see the Appendix, formula 50 (A.5)).

In view of the type of exact solution (2.5) and the ${ }_{52}$ suggestion on the interface configuration, the kinematic ${ }_{53}$ condition is identically satisfied and the normal and ${ }_{54}$ tangential components of the dynamic condition have ${ }_{55}$ the following form

$p_{1}=p_{2}, \quad \rho_{1} \nu_{1} \frac{d u_{1}}{d y}=\rho_{2} \nu_{2} \frac{d u_{2}}{d y}-æ \frac{\partial T}{\partial x}$.

The first equation in (2.11) is the result of the following ${ }^{58}$ relation

$$
-\operatorname{Re}\left(p_{1}-p_{2}\right)+2\left(\frac{d v_{1}}{d y}-\bar{\rho} \bar{\nu} \frac{d v_{2}}{d y}\right)=\frac{1}{\mathrm{Ca}} 2 H \sigma
$$

${ }_{41} \quad \kappa_{1} \frac{\partial T_{1}}{\partial y}-\kappa_{2} \frac{\partial T_{2}}{\partial y}-\left.\delta \kappa_{2} \frac{\partial C}{\partial y}\right|_{y=0}=-L M$.

Here, $\kappa_{j}$ is the thermal conductivity coefficient, $L$ is the 82 latent heat of evaporation, $M$ is the mass flow rate of 83 evaporation defined in the exact mass balance equation (Bekezhanova and Goncharova 2016)

$$
M=-\left.D \rho_{2}\left(\frac{\partial C}{\partial y}+\alpha \frac{\partial T}{\partial y}\right)\right|_{y=0} .
$$

This value is only specified for the determination of the relationship between the thermal and mass balance conditions at the interface. The positive values of $M$ correspond to evaporation, while the negative ones to condensation. Besides, $M$ is an additional quantitative characteristic for comparing the analytical and experimental results. In constructing the solution we realize exactly the case with the constant evaporation mass flow rate $M$. The interest to this situation has been due to the comparison of the quantitative flow characteristics obtained in the experiments (Goncharova et al. 2015), where the experimental data are presented as trendlines.

The saturated vapor concentration can be found with the help of the relation

$\left.C\right|_{y=0}=C_{*}\left[1+\varepsilon\left(\left.T_{2}\right|_{y=0}-T_{0}\right)\right]$.

Here, $\varepsilon=L \mu /\left(R^{*} T_{0}^{2}\right), \mu$ is the molar mass of the evaporating liquid, $R^{*}$ is the universal gas constant, $C_{*}$ is the saturated vapor concentration at $T_{2}=T_{0}$.

To close the problem statement the mass flow rate of the gas is set

$R=\int_{0}^{h_{2}} \rho_{2} u_{2}(y) d y$.

The comprehensive substantiation of using equations (2.1) - (2.4), taking into account the thermal diffusion effects in describing the joint flow of a volatile liquid and gas-vapor mixture, conditions (2.12) and (2.13) was provided in (Bekezhanova and Goncharova 2016).

\subsection{Nondimensionalization way and dimensionless parameters}

Let us introduce non-dimensional variables and functions. We choose $h_{2}$ as the characteristic length scale, $\nu_{2} / h_{2}$ as the velocity scale, $\rho_{2} \nu_{2}^{2} / h_{2}^{2}$ as the pressure scale, and $\vartheta^{+}$as the temperature scale. The vapor concentration function is a non-dimensional one. The units of the physical parameters for the coupled problem are specified based on the characteristic values for the vapor-gas mixture. The dimensionless variables take the following form: $\xi=x / h_{2}, \eta=y / h_{2}$. For each parameter of the medium $\omega_{j}$ the dimensionless analogue $\omega_{j}^{\prime}=\omega_{j} / \omega_{2}$ is introduced. Then, the index $j=1$ corresponds to the domain $-h \leq \eta \leq 0, h=h_{1} / h_{2}$, and $j=2$ is related to the region $0 \leq \eta \leq 1$. 
Table 1 Physical parameters

\begin{tabular}{lll}
\hline Parameter & HFE-7100 & Nitrogen \\
\hline$\rho, \mathrm{kg} / \mathrm{m}^{3}$ & $1.5 \cdot 10^{3}$ & 1.2 \\
$\nu, \mathrm{m}^{2} / \mathrm{s}$ & $0.38 \cdot 10^{-6}$ & $0.15 \cdot 10^{-4}$ \\
$\beta, K^{-1}$ & $1.8 \cdot 10^{-3}$ & $3.67 \cdot 10^{-3}$ \\
$\kappa, \mathrm{W} /(\mathrm{m} \cdot \mathrm{K})$ & 0.07 & 0.02717 \\
$\chi, \mathrm{m}^{2} / \mathrm{s}$ & $0.4 \cdot 10^{-7}$ & $0.3 \cdot 10^{-4}$ \\
$T_{0}, \mathrm{~K}$ & 293.15 & 293.15 \\
$æ, \mathrm{~N} /(\mathrm{m} \cdot \mathrm{K})$ & $1.14 \cdot 10^{-4}$ & \\
$L,(\mathrm{~W} \cdot \mathrm{s}) / \mathrm{kg}$ & $1.11 \cdot 10^{5}$ & \\
$\mu, \mathrm{kg} / \mathrm{mol}$ & 0.25 & \\
$D, \mathrm{~m}^{2} / \mathrm{s}$ & & $0.7 \cdot 10^{-5}$ \\
$\gamma$ & & -0.5 \\
$C_{*}$ & & 0.45 \\
$\delta, \mathrm{K}$ & & $10^{-5}$ \\
$\alpha, \mathrm{K}^{-1}$ & & $5 \cdot 10^{-3}$ \\
\hline
\end{tabular}

In this way the problem under study is characterized ${ }^{40}$ by the following dimensionless parameters and similar- ${ }^{41}$ ity criteria:

$$
\begin{gathered}
\mathrm{Gr}=\frac{g \beta_{2} \vartheta^{+} h_{2}^{3}}{\nu_{2}^{2}}, \operatorname{Pr}=\frac{\nu_{2}}{\chi_{2}}, \mathrm{Ga}=\frac{g h_{2}^{3}}{\nu_{2}^{2}}, \\
\mathrm{Le}=\frac{D}{\chi_{2}}, \quad \mathrm{Q}=\frac{A h_{2}}{\vartheta^{+}} .
\end{gathered}
$$

1 Here, Gr, Pr, Ga, Le are the Grashof, Prandtl, Galileo, ${ }^{46}$ Lewis numbers, $\mathrm{Q}$ is the thermal load parameter.

\section{Influence of the problem parameters on the basic flow structure}

All the investigations are performed for the liquid-gas 55 system like HFE-7100 - nitrogen. The HFE-7100 fluid 56 is a segregated HydroFluoroEther, a dielectric used as 57 a coolant in thermostabilization and liquid cooling sys- 58 tems due to the combination of such properties as volatils9 ity and low surface tension. The parameters character- 60 izing the physico-chemical properties of the substances 61 are given in Table 1. Taking into account the nondi- 62 mensionalization method chosen, the Prandtl and Lewis ${ }^{63}$ numbers are constant: $\operatorname{Pr}=0.5, \mathrm{Le}=0.23$. Thickness 64 of the upper (gas-vapor) layer $h_{2}=5 \mathrm{~mm}$, gas flow 65 rate $R=9.6 \cdot 10^{-6} \mathrm{~kg} /(\mathrm{m} \cdot \mathrm{s})$ and values $\vartheta^{+}=\vartheta^{-}=66$ $293.15 \mathrm{~K}$ are fixed for all the cases under study. The value $g=g_{0}=9.81 \mathrm{~m} / \mathrm{s}^{2}$ corresponds to the conditions ${ }^{67}$ of normal gravity; for this case $\mathrm{Gr}=\mathrm{Gr}_{0}=5863.44,{ }^{68}$ $\mathrm{Ga}=\mathrm{Ga}_{0}=5450$.

\subsection{Gravity effect}

We present the distributions of the basic characteristics 74 of the two-layer flow (longitudinal velocity $u$, temper- 75 ature $T$ and vapor concentration $C$ ) in the horizontal 76

\section{。}

\section{.}

\section{.}

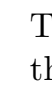

\section{.}

(1) (t) (1)

layer and profiles of the evaporation mass flow rate $M$, which are appropriate to different values of the gravity acceleration $g$. The variations of $g$ correspond to the changes in the Grashof and Galileo numbers. The distributions are defined by solution (2.5).

The hydrodynamic, thermal and vapor concentration fields in the two-layer system with $h_{1}=3 \mathrm{~mm}$, $A_{1}=A_{2}=3 \mathrm{~K} / \mathrm{m}$ in microgravity, terrestrial conditions and hypergravity are shown in Fig. 2. In the cases studied the thermal load parameter $\mathrm{Q}=5.12 \cdot 10^{-5}$, $\mathrm{Gr}=\mathrm{Gr}_{0} \cdot 10^{-2}, \mathrm{Ga}=\mathrm{Ga}_{0} \cdot 10^{-2}$ (Fig. 2(a) $-(\mathrm{c})$ ), $\mathrm{Gr}=\mathrm{Gr}_{0}, \mathrm{Ga}=\mathrm{Ga}_{0}$ (Fig. 2(d)-(f)), Gr $=10 \mathrm{Gr}_{0}$, $\mathrm{Ga}=10 \mathrm{Ga}_{0}$ (Fig. 2(g)-(i)). In weak and normal gravitational fields the typical regime for two-layer flows with a quite small liquid layer thickness is a thermocapillary one. It is characterized by the formation of the reverse flow due to the Marangoni effect causing the liquid motion from the hot pole to the cold one. A thermocline with the maximum temperature is originated near the interface. The heat loss due to evaporation is insignificant and compensated by the supply of warm mass provided by the Marangoni effect. The stable temperature stratification is formed in the liquid phase. In microgravity a pure thermocapillary flow arises in the lower layer, when the fluid moves from the domain with high temperature to the cold region across the entire height of the liquid (Fig. 2(a)-(c)). The basic mechanism generating the flow is a thermocapillary one. Under normal gravity the flow retains predominantly the thermocapillary character, but within the liquid layer the counter current zones appear (Fig. 2(d)-(f)). According to the Napolitano's classification of the flow ypes (Napolitano 1980, Bekezhanova and Goncharova 2016), these patterns are the flows of the mixed type. Two or more rival mechanisms induce the flow. With the increasing gravity action the essential alteration of the velocity and temperature fields occurs. The gravitational effects suppress the impact of the Marangoni forces and the Poiseuille type flow is formed in the system. The interface cooling due to evaporation becomes significant and the unstable temperature stratification occurs in the liquid layer (Fig. 2(g) - (i)).

Under microgravity the unstable temperature stratification in the upper layer is formed and the influence of the convective mechanism in the gas phase is enhanced, the "hot" vapor comes up to the upper boundary and the near-wall concentration increases (Fig. 2(c)). With the increasing gravity it is more difficult for molecules to overcome interparticle attractive forces and to pass into the gaseous state. Therefore, the vapor concentration in the gas drops. Furthermore, under hypergravity in the upper layer the stable temperature strat- 
Table 2 Working ranges of changing $A$ and $\mathrm{Q}$ for different 44 values of $h_{1}$

\begin{tabular}{lll}
\hline$h_{1}, \mathrm{~mm}$ & $A, \mathrm{~K}$ & $\mathrm{Q}$ \\
\hline 2 & {$[1.64 ; 7.02]$} & {$\left[2.8 \cdot 10^{-5} ; 11.97 \cdot 10^{-5}\right]$} \\
3 & {$[0.28 ; 7.83]$} & {$\left[4.7 \cdot 10^{-6} ; 13.36 \cdot 10^{-5}\right]$} \\
4 & {$[-0.92 ; 8.55]$} & {$\left[-1.58 \cdot 10^{-5} ; 14.59 \cdot 10^{-5}\right]$} \\
5 & {$[-1.99 ; 9.19]$} & {$\left[-3.39 \cdot 10^{-5} ; 15.68 \cdot 10^{-5}\right]$} \\
6 & {$[-2.94 ; 9.77]$} & {$\left[-5.02 \cdot 10^{-5} ; 16.66 \cdot 10^{-5}\right]$} \\
\hline
\end{tabular}

ification occurs, and the maximum vapor concentration ${ }^{53}$ is reached near the interface (Fig. 2(i)).

The dependence of the evaporation mass flow rate ${ }^{55}$ $M$ on the temperature gradient $A$ in the gravitational ${ }^{56}$ fields of different intensity is given in Fig. 3(a). The pro- ${ }^{57}$ files 1, 2, 3 are obtained for the system with $h_{1}=3 \mathrm{~mm}^{58}$ and different thermal load applied on the solid walls. ${ }^{59}$ The value of the longitudinal temperature gradient $A^{60}$ on the interface is computed using the formula $(\mathrm{A} .5)^{61}$ with $A_{1}=5 \mathrm{~K} / \mathrm{m}$ and $A_{2}$ varying from -20 to $20 \mathrm{~K} / \mathrm{m}^{62}$ The working ranges of changing $A$ and $\mathrm{Q}$ correspond- ${ }^{63}$ ing to these changes of $A_{2}$ are specified in Table $2 .{ }^{64}$ The interface temperature rises and the number of liq- ${ }^{65}$ uid molecules, whose the kinetic energy is higher than ${ }^{66}$ the average energy, increases due to the thermal mo- ${ }^{67}$ tion. Therefore, with the increasing $A$ the mass of the ${ }^{68}$ evaporating liquid increases. Such a character of the ${ }^{69}$ dependence of $M$ on the thermal load is completely ${ }^{70}$ justified by the experimental data (Lyulin and Kabov ${ }^{71}$ 2013). There exists such a critical thermal load which ${ }^{72}$ alters the qualitative behavior of $M$ to the contrary one. ${ }^{73}$ If $A<A_{c r}$, then gravity impedes evaporation (at the ${ }^{74}$ fixed $A$ the values of $M$ in hypergravity is smaller, than ${ }^{75}$ they are in the terrestrial conditions and microgravity, ${ }^{76}$ compare the curves 1 and 2,2 and 3 in Fig. 3(a)), if ${ }^{77}$ $A>A_{c r}$, then, gravity contributes to the growth of the ${ }^{78}$ evaporation mass flow rate $\left(A_{c r} \approx 5 \mathrm{~K} / \mathrm{m}\right.$, and, hence, ${ }^{79}$ $\left.\mathrm{Q}_{c r} \approx 8.5 \cdot 10^{-5}\right)$ for the system with $\left.h_{1}=3 \mathrm{~mm}\right) .{ }^{80}$ The effect can be explained by the variations in the va- 81 por concentration. The values of $C$ decrease with the 82 increasing gravity (compare Fig. 2(c), (f), (e)). It is 83 known, that the lower is the vapor concentration in the 84 gas flux, the faster is evaporation (Voropai and Shlepov 85 1980). With the growing thermal load the difference in 86 the values of $C$ becomes essential, therefore, at quite ${ }^{87}$ large $A$ the evaporation mass flow rate $M$ under the hy- 88 pergravity conditions is higher than under microgravity. 89 Thus, if $A<A_{c r}$ the thermal effects play a significant 90 role (the higher the temperature, the faster is evapo- 91 ration) and the kinetic mechanism is a leading one. If 92 $A>A_{c r}$, then, in hypergravity the concentration drops 93 and, therefore, the evaporation mass flow rate increases 94 due to the diffusive mechanism.
3.2 Impact of the liquid layer thickness

Figure 4 presents possible distributions of the velocity and temperature under the microgravity conditions at $g=g_{0} \cdot 10^{-1}\left(\mathrm{Gr}=\mathrm{Gr}_{0} \cdot 10^{-1}, \mathrm{Ga}=\mathrm{Ga}_{0} \cdot 10^{-1}\right)$ and $A_{1}=A_{2}=A=3 \mathrm{~K} / \mathrm{m}$ with various thicknesses of the liquid layer. In weak gravitational fields the basic flow is the thermocapillary one in the entire range of the liquid layer thickness values under consideration. The reverse flows induced by the Marangoni effect are formed in the bottom layer and a thermocline with the maximum temperature appears near the interface. The investigations of the flow features on the basis of exact solution (2.5) allows one to conclude that at the fixed positive thermal load given by $A_{1}=A_{2}$, further growth of $h_{1}$ always leads to the change of the flow type from the thermocapillary to the mixed one. This is due to the diminution of the Marangoni forces with the increase of the liquid thickness. In the case of equal thermal load the influence of the thermocapillary effect with small $g$ is essential to form the patterns of the reverse flows even with small positive $A_{j}$ (the thermal effects in the system were investigated in (Rezanova and Shefer 2017)). The increase of the gas flow rate allows one to control the flow as well, at fixed $h_{1}$ and $A$ there exists a value of $R$ such that the flow pattern is of the mixed or Poiseuille's type (Bekezhanova and Goncharova 2016).

It should be noted, that the solution under study does not adequately describe evaporative convection in the system being under the hypergravity conditions with quite a large liquid layer thickness. For the working media used HFE-7100 - nitrogen solution (2.5) predicts nonphysical (infeasible) values of the vapor concentration function and non-typical temperature drop in the channel for $h_{1}=4,5,6 \mathrm{~mm}$. The result confirms the conclusion concerning the boundaries of applicability of the Birikh type solution obtained by Shliomis and Yakushin (1972).

The variations of $h_{1}$ lead to the changes in the values of $A$, and, hence, of $\mathrm{Q}$, in the case if $A_{1} \neq A_{2}$ (see formula (A.5)). The variations of the evaporation mass flow rate $M$ with the changing $h_{1}$ and $A$ are presented in Fig. 3(b) for microgravity and in Fig. 3(c) for the terrestrial conditions. As earlier, the profiles $M(A)$ are obtained for the system subjected to different thermal loads applied to the solid walls. The value of the longitudinal temperature gradient $A$ on the interface is computed using the formula (A.5) with $A_{1}=5 \mathrm{~K} / \mathrm{m}$ and $A_{2}$ varying from -20 to $20 \mathrm{~K} / \mathrm{m}$. Corresponding ranges of changing $A$ and $\mathrm{Q}$ for different thicknesses of the liquid layer $h_{1}$ are presented in Table 2.

The qualitative changes of the mass transfer processes are observed with the increasing height of the 


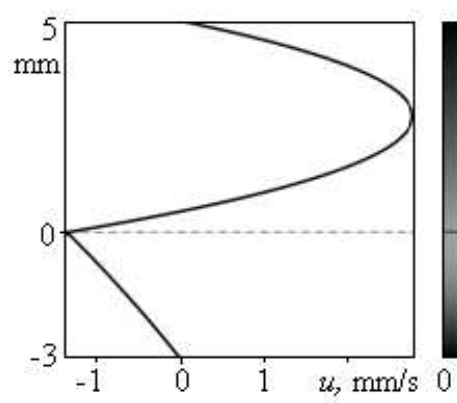

(a)

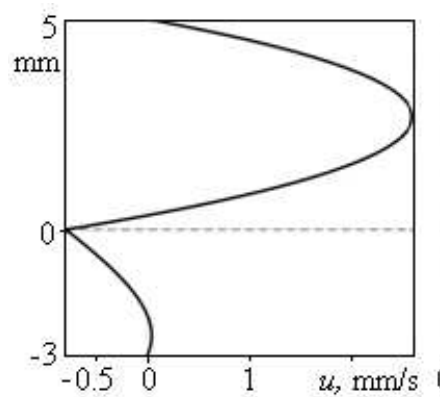

(d)

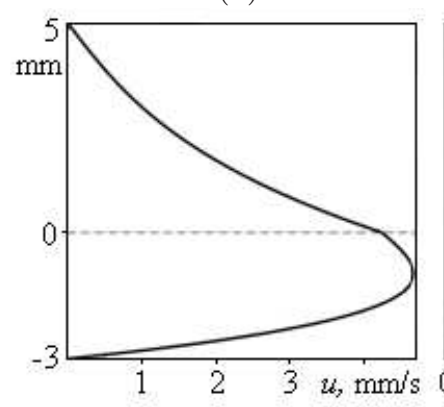

(g)

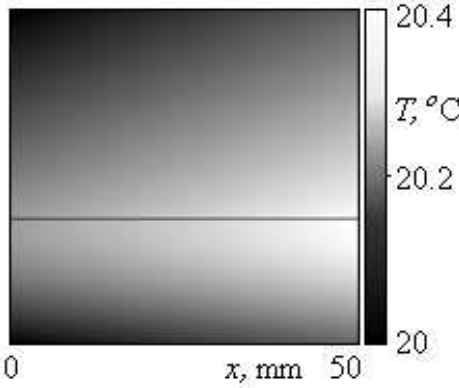

(b)

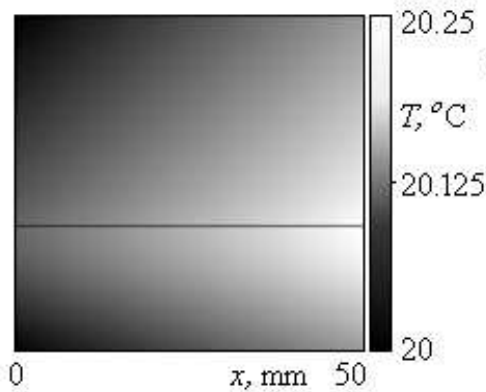

(e)

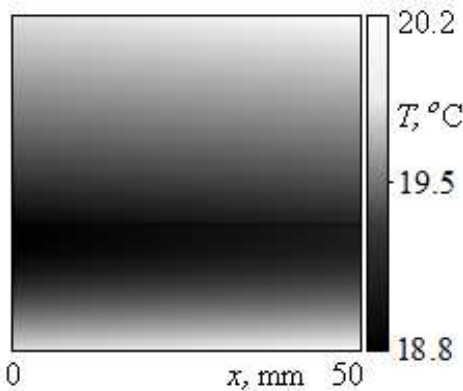

(h)

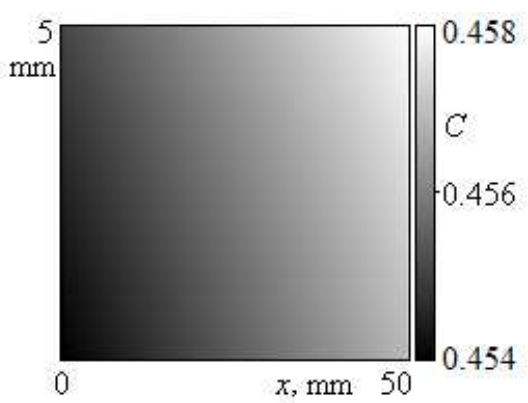

(c)

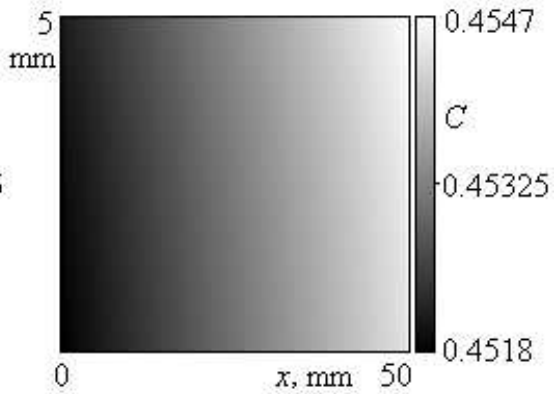

(f)

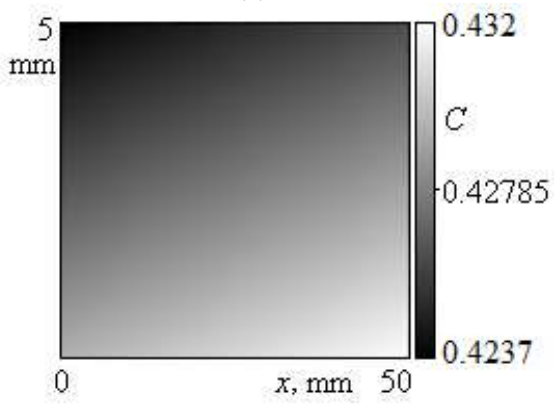

(i)

Fig. 2 Distributions of the longitudinal velocity $u(y)(\mathrm{a}, \mathrm{d}, \mathrm{g})$, temperature $T(x, y)(\mathrm{b}, \mathrm{e}, \mathrm{h})$ and vapor concentration $C(x, y)$ $(\mathrm{c}, \mathrm{f}, \mathrm{i})$ in the system with $h_{1}=3 \mathrm{~mm}, A_{1}=A_{2}=3 \mathrm{~K} / \mathrm{m}$ in microgravity $\left(g=g_{0} \cdot 10^{-2}(\mathrm{a}-\mathrm{c})\right)$, terrestrial conditions $\left(g=g_{0}\right.$, $(\mathrm{d}-\mathrm{f}))$, and hypergravity $\left(g=10 g_{0},(\mathrm{~g}-\mathrm{i})\right)$

1 liquid layer. At $h_{1}=2 \mathrm{~mm}$ and $h_{2}=4 \mathrm{~mm}$ in the 19 temperature range only evaporation $(M>0)$ occurs 20 (curves 1 and 2 in Fig. 3(b), (c)), but at $h_{1}=6 \mathrm{~mm}$ the 21 negative temperature gradients originate on $\Gamma$ and va- 22 por condensation $(M<0)$ begins (curve 3 in Fig. 3(b), 23 (c)). Thus, under the same conditions (thermal load 24 and gravity action) applied to the systems with various 25 thicknesses of the liquid layer the mass of the evapo- 26 rating liquid is different. With the increasing height $h_{127}$ the evaporation mass flow rate $M$ drops considerably. 28 Such a character of the dependence of $M$ on the liquid ${ }_{29}$ thickness is confirmed by the experimental data (Lyulin 30 and Kabov 2013). In the experiments it was found that 31 at the fixed thermal load there existed the local maxi- 32 mum of $M$ reached at some values of $h_{1}$. One can see 33 that for the system with $h_{1}=2 \mathrm{~mm}$ the local maxi- 34 mum is achieved at $A=3.8 \mathrm{~K} / \mathrm{m}\left(\mathrm{Q}=6.48 \cdot 10^{-5}\right)$ in $_{35}$ microgravity $\left(\mathrm{Gr}=\mathrm{Gr}_{0} \cdot 10^{-2}\right)$ and at $A=3.93 \mathrm{~K} / \mathrm{m}$
$\left(\mathrm{Q}=6.7 \cdot 10^{-5}\right)$ under the terrestrial conditions $(\mathrm{Gr}=$ $\mathrm{Gr}_{0}$ ). Under these circumstances (at the given values of $A, g$ and $h_{1}$ ) vapor becomes saturated and evaporation ceases. With the increasing thermal load the local maximum is achieved at larger values of $h_{1}$ (Lyulin and Kabov 2013). If the liquid layer decreases in thickness, then its volumetric energy decreases, but the surface energy, which is axiomatically identified with the surface tension coefficient $\sigma(T)$, becomes the main driving factor. It leads to the molecules within the superficial thin layer being more strongly attracted to each other and the surface tension forces being more intensive. The forces work positively to transfer the molecules from the volume phase to the surface one, therefore, the thin liquid layer evaporates easier than the layer with larger thickness. Thus, the functions $M(A)$ obtained on the basis of exact solution (2.5) are physically plausible. 


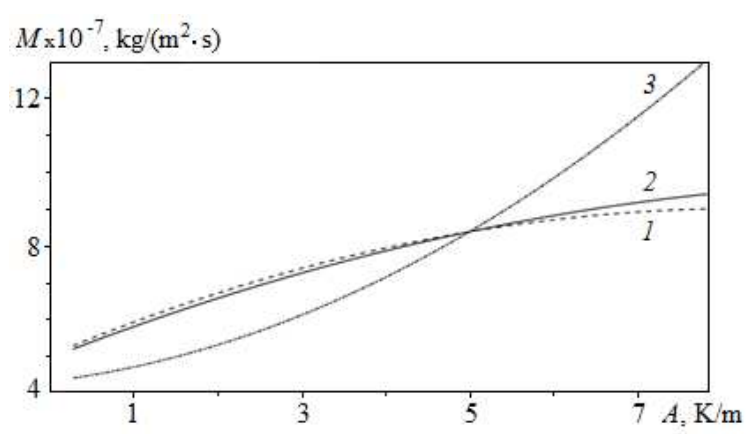

(a)

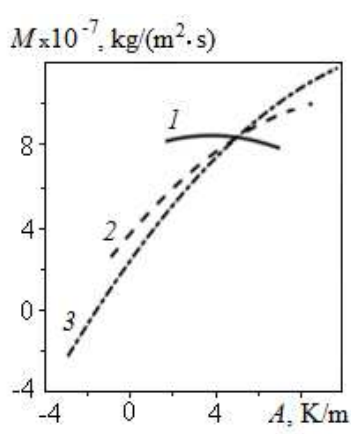

(b)

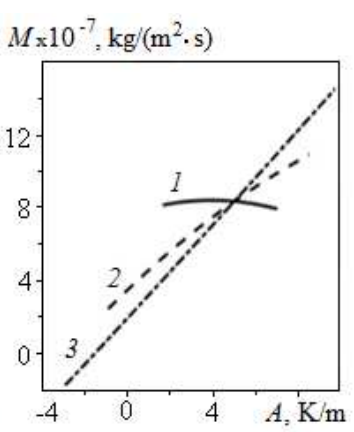

(c)

Fig. 3 Evaporation mass flow rate $M(A)$ : (a) $-h_{1}=3 \mathrm{~mm}, 1-g=g_{0} \cdot 10^{-2}, 2-g=g_{0}, 3-g=10 g_{0} ;(\mathrm{b})-$ $g=g_{0} \cdot 10^{-2}, 1-h_{1}=2 \mathrm{~mm}, 2-h_{1}=4 \mathrm{~mm}, 3-h_{1}=6 \mathrm{~mm} ;(\mathrm{c})-g=g_{0}, 1-h_{1}=2 \mathrm{~mm}, 2-h_{1}=4 \mathrm{~mm}, 3-$ $h_{1}=6 \mathrm{~mm}$

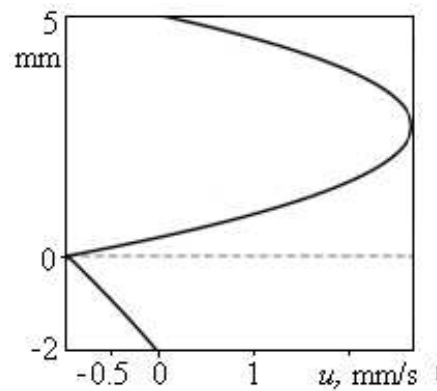

(a)

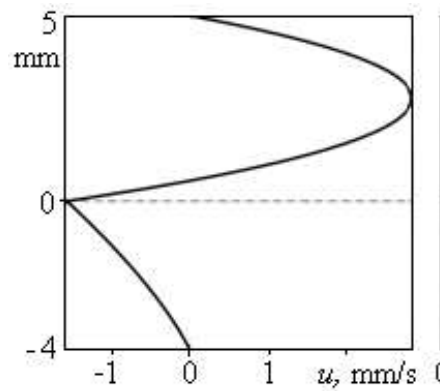

(d)

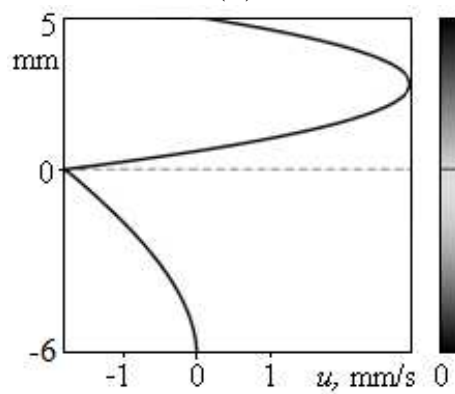

(g)

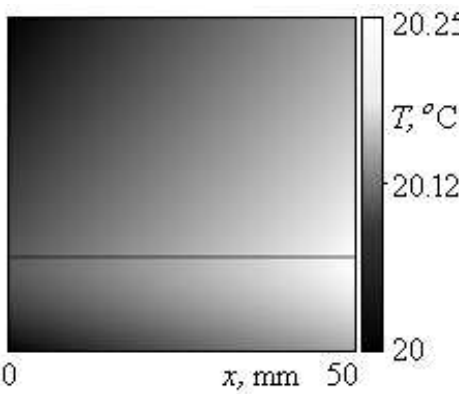

(b)

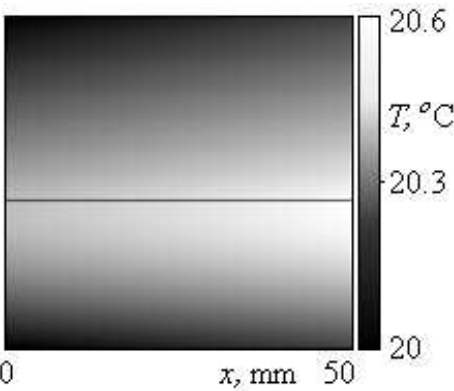

(e)

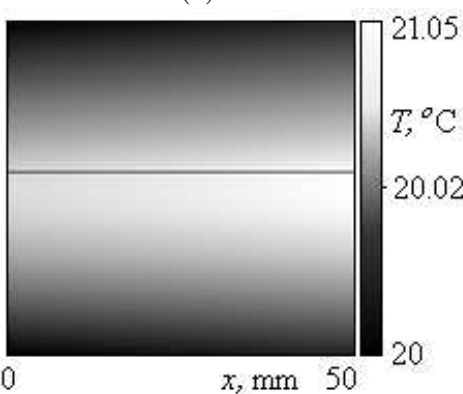

(h)

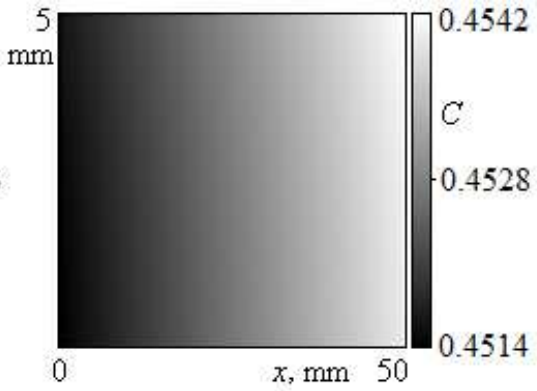

(c)

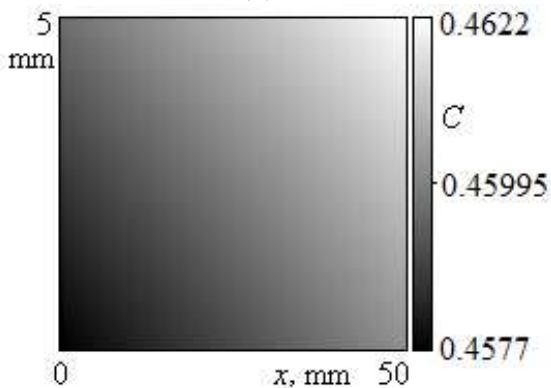

(f)

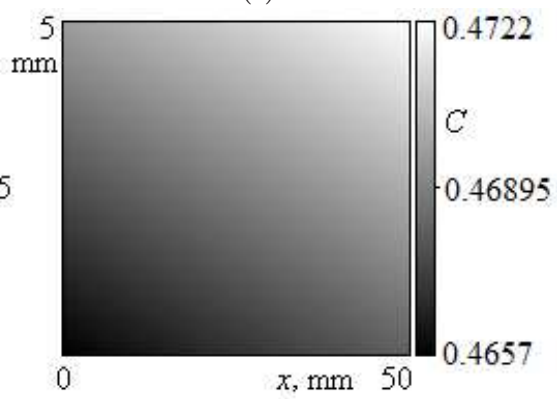

(i)

Fig. 4 Distributions of the longitudinal velocity $u(y)(\mathrm{a}, \mathrm{d}, \mathrm{g})$, temperature $T(x, y)(\mathrm{b}, \mathrm{e}, \mathrm{h})$ and vapor concentration $C(x, y)$ $(\mathrm{c}, \mathrm{f}, \mathrm{i})$ in the system with $A_{1}=A_{2}=3 \mathrm{~K} / \mathrm{m}$ under microgravity $\left(g=g_{0} \cdot 10^{-1}\right)$ at $h_{1}=2 \mathrm{~mm}(\mathrm{a}-\mathrm{c}), h_{1}=4 \mathrm{~mm}(\mathrm{~d}-\mathrm{f})$, $h_{1}=6 \mathrm{~mm}(\mathrm{~g}-\mathrm{i})$ 
3.3 Maps of the basic flow types

We present diagrams of regimes in the parameter space ${ }_{53}$ (Q, Gr) for the geometrical configurations with $h_{1}=2_{54}$ and $3 \mathrm{~mm}$ in Fig. 5, and for $h_{1}=4,5$ and $6 \mathrm{~mm}$ in ${ }_{55}$ Fig. 6. Zones 1, 2, 3 correspond to the Poiseuille type ${ }_{56}$ flow, to the mixed type flow and to the pure thermocapillary flow, respectively. Ranges of $\mathrm{Q}$ variations in the figures are chosen in accordance with Table 2. Working range of $\mathrm{Gr}$ in Fig. 6 does not include values of the Grashof number corresponding to the hypergravity conditions (Sec. 3.2)

Typical feature for systems with thin liquid layer under study is the formation of the pure thermocapillary flows in the whole range of the thermal load in the microgravity conditions (Fig. 5(a)). With increasing $h_{1}$ the Marangoni effect wanes, and the pure thermocapillary flow is observed under quite large positive thermal load (Fig. 6). Thus, zones 3 correspond to the domains of $\mathrm{Q}$ and $\mathrm{Gr}$ values, in which the thermocapillary mechanism dominates. Regions of formation of the mixed type flows (zones 2) satisfy the conditions of coexistence of convective and thermocapillary mechanisms. Predominance of the gravitational effects appears in zones 1 and it is mainly evident under hypergravity (Fig. 5), in the case of negative or weak positive thermal load or in the system with $h_{1}=4$ and $5 \mathrm{~mm}$ (Fig. 6).

Here we also specify ranges of non-dimensional parameters at which the constructed stationary exact solution adequately describes real flows in the system "HFE-7100-nitrogen" with mass transfer across the interface and predicts feasible values of the vapor con- 59 centration, velocity and temperature of both media: for 60 $h_{1}=2,3 \mathrm{~mm} \mathrm{Gr} \in[58.63 ; 58634.4]$, for $h_{1}=4,5,6 \mathrm{~mm}$ $\mathrm{Gr} \in[58.63 ; 5863.44]$ and $\mathrm{Ma} \in[-105.5 ; 105.5]$ for all $_{61}$ the thicknesses of the liquid layer under consideration. in a system of equations for the amplitudes of small disturbances. The system in the dimensionless form is written as follows (the primes for the non-dimensional parameters and functions will be omitted henceforth):

$$
\begin{aligned}
& -h<\eta<0: \quad-i \lambda U_{1}+i \alpha_{x} u_{1} U_{1}+u_{1}^{\prime} V_{1}= \\
& =-\frac{i \alpha_{x}}{\rho} P_{1}+\nu\left(U_{1}^{\prime \prime}-\alpha_{x}^{2} U_{1}\right), \\
& -i \lambda V_{1}+i \alpha_{x} u_{1} V_{1}= \\
& =-\frac{1}{\rho} P_{1}^{\prime}+\nu\left(V_{1}^{\prime \prime}-\alpha_{x}^{2} V_{1}\right)+\beta \mathrm{Gr} \Theta_{1}, \\
& i \alpha_{x} U_{1}+V_{1}^{\prime}=0, \\
& -i \lambda \Theta_{1}+i \alpha_{x} u_{1} \Theta_{1}+U_{1} T_{1 \xi}+V_{1} T_{1 \eta}= \\
& =\frac{\chi}{\operatorname{Pr}}\left(\Theta_{1}^{\prime \prime}-\alpha_{x}^{2} \Theta_{1}\right), \\
& 0<\eta<1: \quad-i \lambda U_{2}+i \alpha_{x} u_{2} U_{2}+u_{2}^{\prime} V_{2}= \\
& =-i \alpha_{x} P_{2}+U_{2}^{\prime \prime}-\alpha_{x}^{2} U_{2}, \\
& -i \lambda V_{2}+i \alpha_{x} u_{2} V_{2}= \\
& =-P_{2}^{\prime}+V_{2}^{\prime \prime}-\alpha_{x}^{2} V_{2}+\mathrm{Gr} \Theta_{2}+\gamma \mathrm{Ga} S, \\
& i \alpha_{x} U_{2}+V_{2}^{\prime}=0, \\
& -i \lambda \Theta_{2}+i \alpha_{x} u_{2} \Theta_{2}+U_{2} T_{2 \xi}+V_{2} T_{2 \eta}= \\
& =\frac{1}{\operatorname{Pr}}\left[\Theta_{2}^{\prime \prime}-\alpha_{x}^{2} \Theta_{2}+\frac{\delta}{\vartheta+}\left(C^{\prime \prime}-\alpha_{x}^{2} C\right)\right] \\
& -i \lambda S+i \alpha_{x} u_{2} S+U_{2} C_{\xi}+V_{2} C_{\eta}= \\
& =\frac{\mathrm{Le}}{\operatorname{Pr}}\left(S^{\prime \prime}-\alpha_{x}^{2} S+\alpha \vartheta^{+}\left(\Theta^{\prime \prime}-\alpha_{x}^{2} \Theta\right)\right) .
\end{aligned}
$$

The following conditions for the perturbation amplitudes are imposed on the solid walls and interface:

$$
\begin{aligned}
& \eta=-h: \quad U_{1}=V_{1}=\Theta_{1}=0, \\
& \eta=1: \quad U_{2}=V_{2}=\Theta_{2}=S^{\prime}=0 . \\
& \eta=0: \quad U_{1}=U_{2}, \quad V_{1}=V_{2}=0, \quad \Theta_{1}=\Theta_{2}, \\
& U_{2}^{\prime}-\nu \rho U_{1}^{\prime}+i \alpha_{x}\left(V_{2}-V_{1}\right)=\frac{\mathrm{Ma}}{\mathrm{Q}} i \alpha_{x} \Theta, \\
& P_{1}-P_{2}=2\left(\nu \rho V_{1}^{\prime}-V_{2}^{\prime}\right), \\
& k \Theta_{1}^{\prime}-\Theta_{2}^{\prime}-\frac{\delta}{\vartheta^{+}} S^{\prime}=\frac{D L \rho_{2}}{k_{2} \vartheta^{+}}\left(S^{\prime}+\alpha \vartheta^{+} \Theta^{\prime}\right) .
\end{aligned}
$$

Here, $\Theta$ is the common temperature value of both media on $\Gamma\left(\Theta=\Theta_{j}, j=1,2\right)$, Ma $=æ A h_{2}^{2} /\left(\nu_{2}^{2} \rho_{2}\right)$ is the Marangoni number.

Now, in $(4.1)-(4.4)$ the prime denotes differentiation concerning the variable $\eta$. In deriving the conditions on $\Gamma$ one assumes that the interface remains nondeformed, i. e. the perturbations of the desired functions do not lead to the interface perturbations. Problem $(4.1)-(4.4)$ is the spectral one for the decrement $\lambda$, 


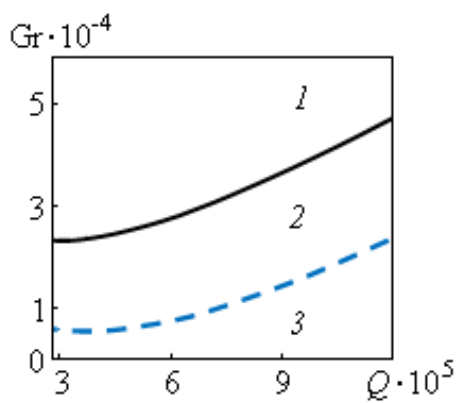

(a)

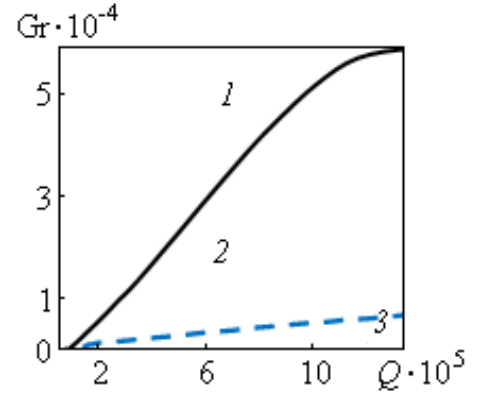

(b)

Fig. 5 Maps of the basic flow types depending on the values of Q and Gr for systems with $h_{1}=2 \mathrm{~mm}(\mathrm{a})$ and $h_{1}=3 \mathrm{~mm}$ (b). Solid line separates zones of the Poiseuille type flows (zone 1) and the mixed type flows (zone 2), dashed line separates zones of the mixed type flows and the pure thermocapillary flows (zone 3)

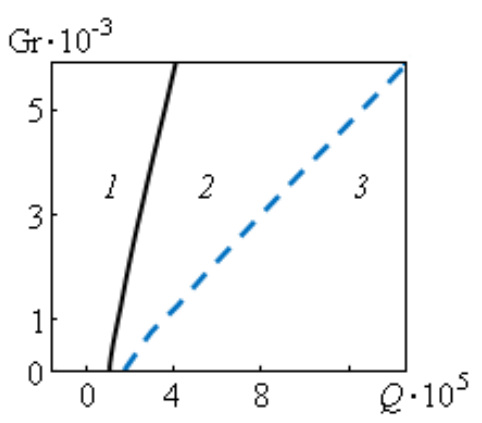

(a)

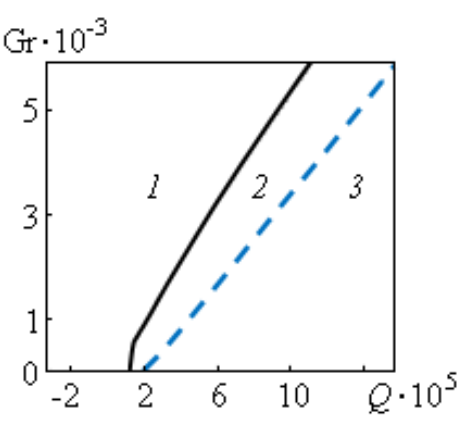

(b)

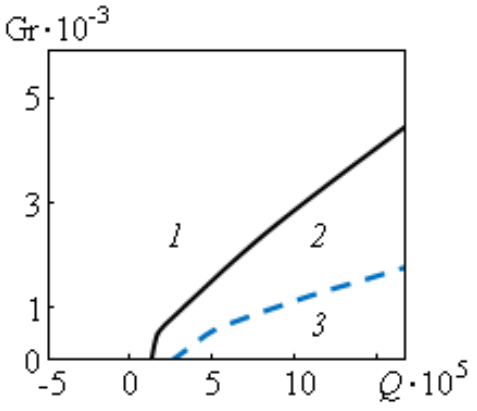

(c)

Fig. 6 Maps of the basic flow types depending on the values of $\mathrm{Q}$ and $\mathrm{Gr}$ for systems with $h_{1}=4 \mathrm{~mm}(\mathrm{a}), h_{1}=5 \mathrm{~mm}(\mathrm{~b})$, $h_{1}=6 \mathrm{~mm}$ (c). Solid line separates zones of the Poiseuille type flows and the mixed type flows, dashed line separates zones of the mixed type flows and the pure thermocapillary flows

the perturbation amplitudes are the unknown functions 20 and define the characteristic perturbations of the nor- 21 mal wave type. If $\lambda_{i}>0$, the disturbances will grow and 22 solution $(2.5)$ will be unstable as related to the normal 23 mode. If $\lambda_{i}=0$ and $\lambda_{r} \neq 0$, neutral oscillations leading ${ }_{24}$ to the formation of oscillating structures will arise in 25 the system.

To obtain the solution of the spectral problem, the ${ }^{27}$ orthogonalization method (Godunov 1961) was mod- 28 ified for solving the problem in the domain with the ${ }^{29}$ interface.

\section{Maps of instability}

\subsection{Critical characteristics of the linear stability}

The main problem is determining the critical thermal loads applied to the external rigid boundaries of the 39 channel and specifying the type of the most dangerous 40 perturbations leading to the stability loss depending 41 on the intensity of the gravity action and liquid layer 42 thickness.
In Figure 7 the neutral curves $A\left(\alpha_{x}\right)$ are presented for the systems with various values of the liquid layer $h_{1}$ depending on the intensity of the gravity action. The values of $A$ lying on the curves define the critical thermal load, at which the two-layer flow loses the stability. The instability domains are denoted by $\mathrm{U}_{m}$, $m=1,2,3$, the subscript $m$ corresponds to the curve number, and all the regions lie to the right of the curves.

It can be seen that for all the configurations under study there exists a thermal load at which the basic flow loses the stability. It should be noted that the convective flows with evaporation are unstable regarding the shortwave perturbations with any values of $A$. In the systems of the two-layer fluids without evaporation the convective flows induced by the joint action of the longitudinal temperature gradients and pressure gradient can be stable concerning the shortwave disturbances in some range of thermal loads (Bekezhanova 2011, 2012). Thus, the mass transfer due to evaporation/condensation has a destabilizing effect.

In weak gravitational field, as described above, the stable temperature stratification is formed owing to the thermocapillary effect not only in the system with small $h_{1}$ (see Fig. 4). This effect stabilizes the flow in the 


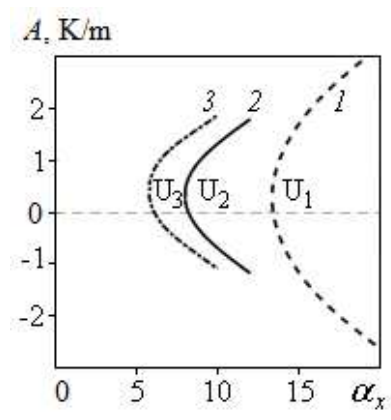

(a)

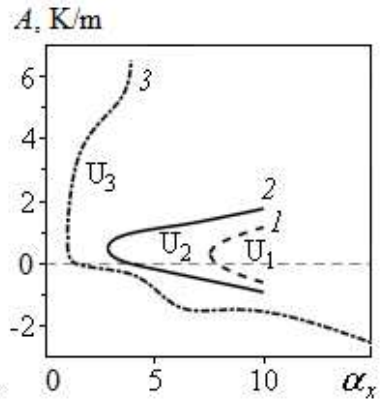

(b)

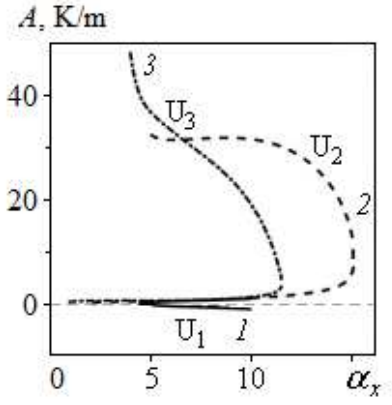

(c)

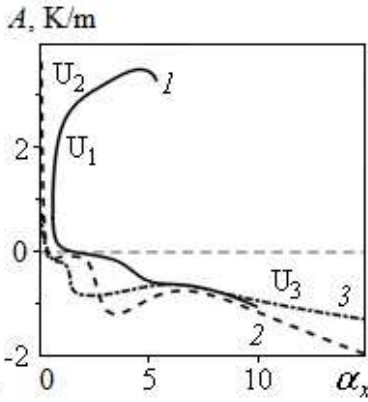

(d)

Fig. 7 Neutral curves $A\left(\alpha_{x}\right)$ : (a) - $h_{1}=2 \mathrm{~mm}, 1-g=g_{0} \cdot 10^{-1} \mathrm{~m} / \mathrm{s}^{2} ; 2-g=g_{0} \mathrm{~m} / \mathrm{s}^{2} ; 3-g=2 g_{0} \mathrm{~m} / \mathrm{s}^{2} ;(\mathrm{b})-$ $h_{1}=3 \mathrm{~mm}, 1-g=g_{0} \cdot 10^{-1} \mathrm{~m} / \mathrm{s}^{2} ; 2-g=g_{0} \mathrm{~m} / \mathrm{s}^{2} ; 3-g=2 g_{0} \mathrm{~m} / \mathrm{s}^{2} ;(\mathrm{c})-g=g_{0} \cdot 10^{-1}, 1-h_{1}=4 \mathrm{~mm} ; 2-$ $h_{1}=5 \mathrm{~mm} ; 3-h_{1}=6 \mathrm{~mm} ;(\mathrm{d})-g=g_{0}, 1-h_{1}=4 \mathrm{~mm} ; 2-h_{1}=5 \mathrm{~mm} ; 3-h_{1}=6 \mathrm{~mm}$

configurations under study. With the increasing grav- 36 ity action the Marangoni effect abates and the instabil- ${ }_{37}$ ity domain expands (compare the regions $\mathrm{U}_{1}, \mathrm{U}_{2}$ and ${ }_{38}$ $\mathrm{U}_{3}$ in Fig. 7(a), (b)). The same pattern of changes of 39 the flow characteristics occurs with the growth of the 40 liquid layer thickness, the stabilizing influence of the thermocapillary effect weakens and the instability zone ${ }^{41}$ is also enlarged (compare the regions $\mathrm{U}_{1}, \mathrm{U}_{2}$ and $\mathrm{U}_{3}$ in ${ }^{42}$ Fig. 7(c), (d)).

\subsection{Selection of modes and typical forms of the most dangerous perturbations}

With the varying $g$ and $h_{1}$ values we observe the change ${ }^{49}$ of the most dangerous disturbance type. Furthermore, ${ }^{50}$ the perturbation pattern can depend on the sign of $A .^{51}$ In fact, the positive values of the longitudinal temper- ${ }^{52}$ ature gradient correspond to heating walls in the di- ${ }^{53}$ rection of the basic flow, and the negative values are ${ }^{54}$ related to cooling walls. The type of the basic flow and ${ }^{55}$ structure of the temperature field can be changed de- ${ }^{56}$ pending on the character of the thermal load (Bekezha- ${ }^{57}$ nova and Goncharova 2016, Bekezhanova et al. 2017). ${ }^{58}$ Therefore, the mechanisms of instability can signifi- ${ }^{59}$ cantly differ in these two cases. Let us denote the insta- ${ }^{60}$ bility domains corresponding to the positive and nega- ${ }^{61}$ tive values of $A$ by $\mathrm{U}^{+}$and $\mathrm{U}^{-}$, respectively.

In Fig. 8 possible forms of the characteristic pertur- ${ }^{63}$ bations arising in the systems under consideration are ${ }^{64}$ presented. The following types of the disturbances are ${ }^{65}$ specified: $(I)$ purely thermocapillary structures with 66 the chessboard pattern of thermal spots (Fig. 8(a)), 67 (II) thermocapillary structures (Fig. 8(b)), (III) mixed 68 type structures with interfacial thermal cores 69 (Fig. 8(c)), (IV) deformed mixed type structures with 70 the chessboard pattern of thermal spots (Fig. 8(d)), 71 $(V)$ mixed type structures with interior located ther- 72 mal cores (Fig. 8(e)), (VI) deformed near-surface vortex structures (Fig. 8(f)), (VII) twin vortex structures (Fig. 8(g)), (VIII) convective cells (Fig. 8(h)), $(I X)$ mixed type structures with double thermal spots (Fig. 8(i)).

The formation of each type of the structures is defined by different mechanisms or their interaction with the basic flow. Patterns $I$ arise in the systems with the stable temperature stratification in the liquid layer or with the thermocline in the liquid. In the latter case the upper part of the liquid layer (over the thermocline) is steadily stratified, and the bottom part (under the thermocline) is gravitationally unstable. The basic mechanism is the thermocapillary effect resulting in the near-surface motion and formation of the near-surface vortices. Let us specify the ranges and values of defining non-dimensional parameters, where this instability mode occurs. It should be noted, that the range of the Marangoni number variations changes with wave number $\alpha_{x}$. In the system with $h_{1}=2 \mathrm{~mm}$ at $\mathrm{Gr}=\mathrm{Gr}_{0}$. $10^{-1}$ under $\alpha_{x}=15$ the Marangoni number variation range is $\mathrm{Ma} \in[-12.42 ; 17.98]$, if $\alpha_{x}=16$, then Ma $\in$ $[-16.47 ; 22.08]$. With further increase in $\alpha_{x}$ the instability domain is widen (see Fig. 7(a), domain $\mathrm{U}_{1}$ ). Under $\alpha_{x}=20$ structures $I$ will appear if the Marangoni number varies in range $\mathrm{Ma} \in[-27.86 ; 33.76]$. In the systems with $h_{1}=5 \mathrm{~mm}$ and $h_{1}=6 \mathrm{~mm}$ the instability mode occurs at $\mathrm{Gr}=\mathrm{Gr}_{0}$, Ma $\in[-20.81 ; 0]$ and $\mathrm{Ma} \in[-13.7 ; 10.7]$, respectively, for all the values of $\alpha_{x}$ under consideration.

Structures II can appear in the systems with the thin liquid layer both in the case of weak unstable stratification and in the steadily stratified state. In the first case the weak convective motion is suppressed by the Marangoni effect, but the convective mechanism can encourage the motion throughout the height of the liquid layer. Upon that, the cores of the arising vor- 


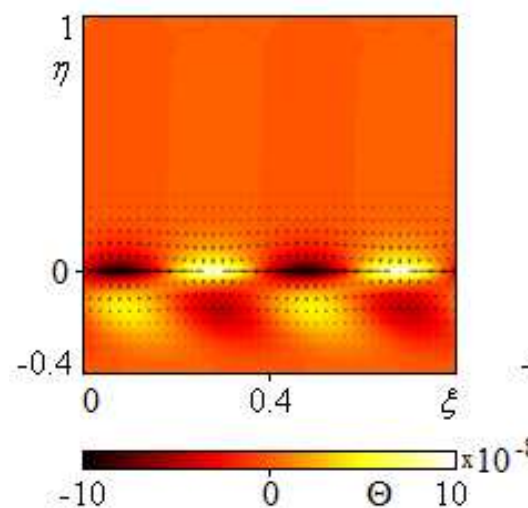

(a)
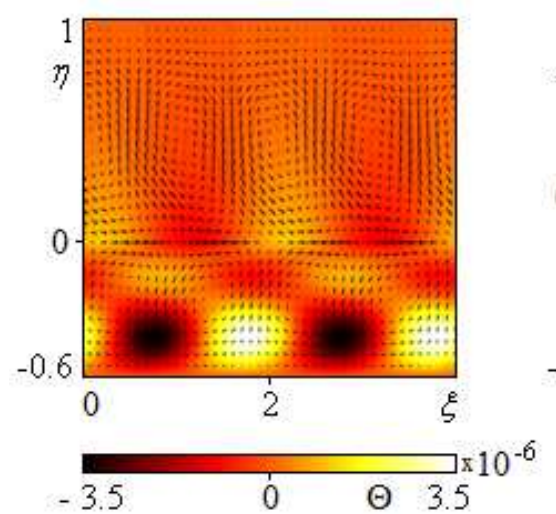

(d)

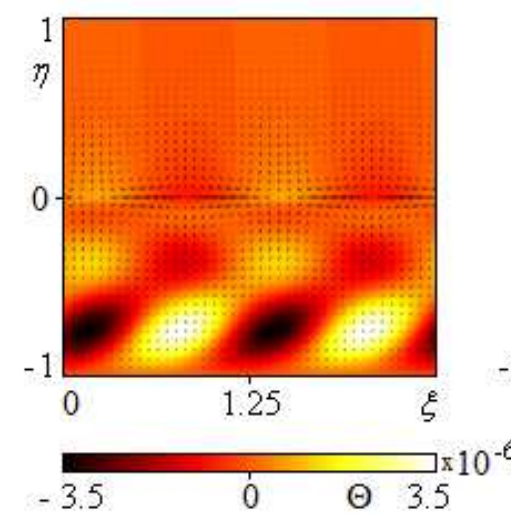

(g)

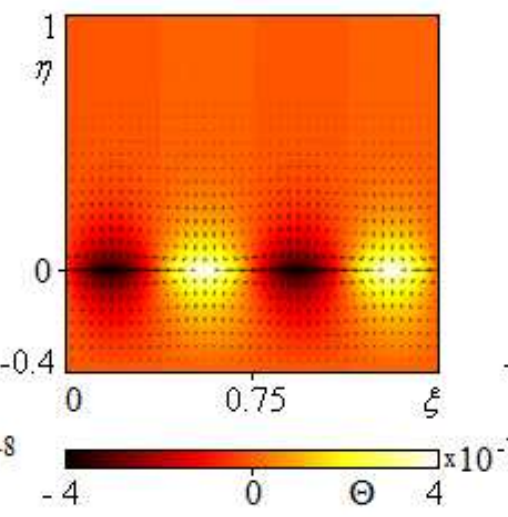

(b)

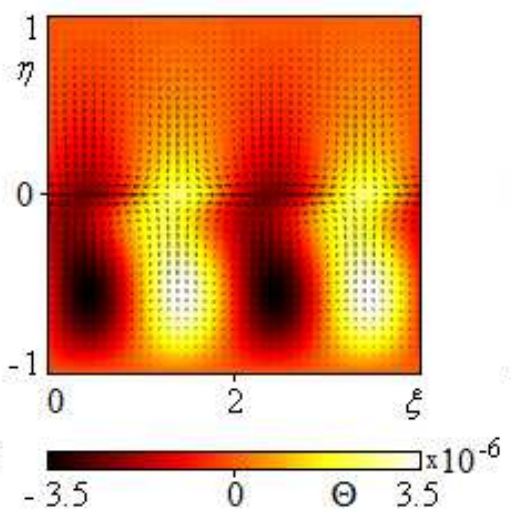

(e)

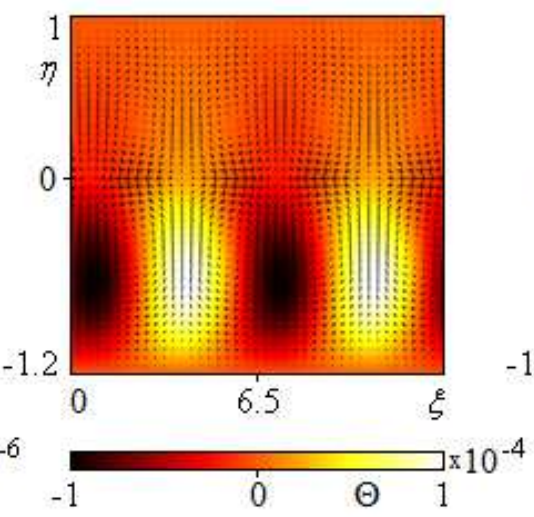

(h)

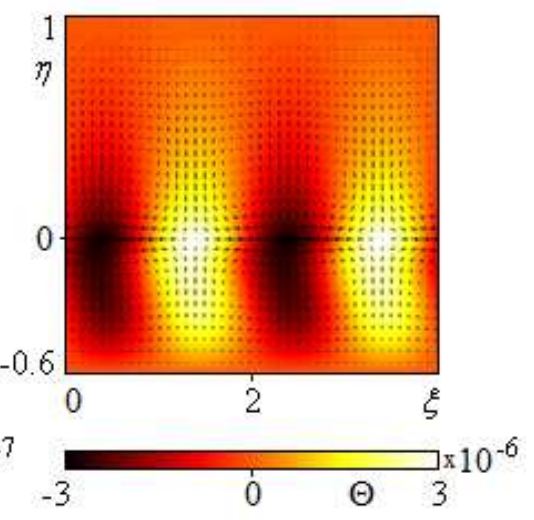

(c)

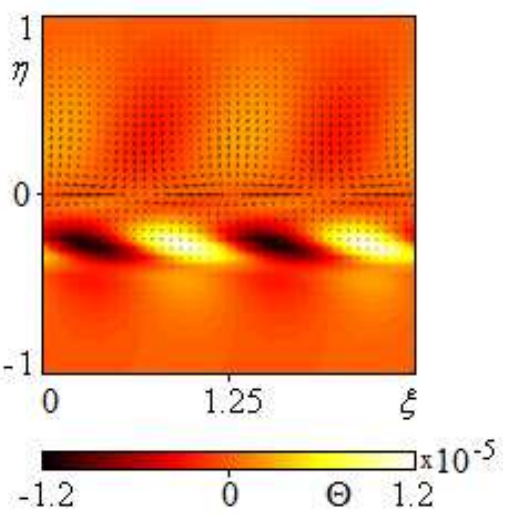

(f)

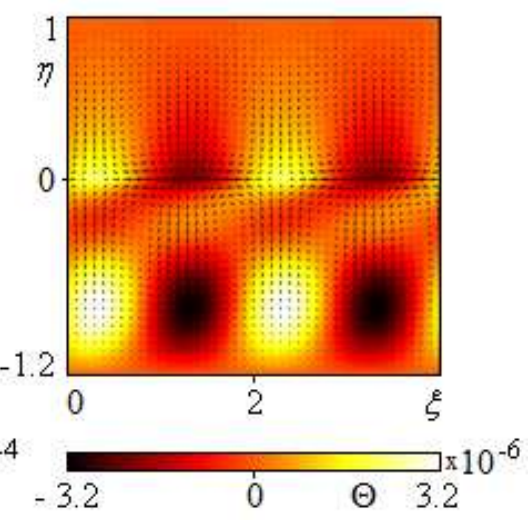

(i)

Fig. 8 Typical patterns of the characteristic perturbations $A\left(\alpha_{x}\right)$ : (a) — purely thermocapillary structures $I$; (b) - thermocapillary structures $I I$; (c) — mixed type structures $I I I$; (d) - deformed mixed type structures $I V$; (e) - mixed type structures $V ;$ (f) — deformed near-surface vortex structures $V I ;(\mathrm{g})$ - twin vortex structures $V I I$; (h) — convective cells $V I I I$; (i) - mixed type structures $I X$

tices are slightly shifted from the interface. Taking into 8 account the primary influence of the thermocapillary 9 effect, structures II are the patterns of the thermo- 10 capillary type. This instability mode arises in the sys- 11 tems with $h_{1}=2 \mathrm{~mm}$ at $\mathrm{Gr}=\mathrm{Gr}_{0}$ and $\mathrm{Gr}=2 \mathrm{Gr}_{0},{ }_{12}$ the Marangoni number variation range also depends 13 on wave number. In the terrestrial conditions $\mathrm{Ma} \in \in_{14}$
$[2.7 ; 4.08]$ under $\alpha_{x}=8$, Ma $\in[-4.11 ; 10.76]$ under $\alpha_{x}=9$, Ma $\in[-7.32 ; 13.85]$ under $\alpha_{x}=10$. In the hypergravity conditions Ma $\in[-3.92 ; 12.4]$ under $\alpha_{x}=7$, $\mathrm{Ma} \in[-6.71 ; 15.04]$ under $\alpha_{x}=8, \mathrm{Ma} \in[-9.15 ; 17.32]$ under $\alpha_{x}=9$. Further increase in $\alpha_{x}$ leads to change of instability type both under normal and above-normal gravity. In the system with $h_{1}=3 \mathrm{~mm}$ patterns $I I$ will 
appear at $\mathrm{Gr}=\mathrm{Gr}_{0} \cdot 10^{-1}$, if Ma $\in[-0.65 ; 6.85]$ un- 54 $\operatorname{der} \alpha_{x}=8$, Ma $\in[-4.05 ; 9.94]$ under $\alpha_{x}=9$, and at 55 $\mathrm{Gr}=2 \mathrm{Gr}_{0}$, if $\mathrm{Ma} \in[-1.14 ; 0]$ under $\alpha_{x}=2, \mathrm{Ma} \in{ }_{56}$ $[-8.48 ; 0]$ under $\alpha_{x}=5$. Further increase in $\alpha_{x}$ also 57 results in change of instability mode regardless of the 58 gravity intensity.

The formation of large scale patterns $I I I$ is induced 60 by the combined action of the thermocapillary and con- 61 vective mechanisms in the systems with the weakly un- 62 steady stratified liquid. The first of them leads to the 63 motion near the interface and this motion is intensi- 64 fied by the convective mechanism. The arising vortex 65 structures have cores which are remote from the in- 66 terface within the liquid layer. The predominant in- 67 fluence of the Marangoni effect is retained under the 68 formation of the patterns. The structures are not ob- 69 served in the system with quite a large liquid layer 70 thickness. Instability mode $I I I$ occurs in the systems 71 with $h_{1}=3 \mathrm{~mm}$ at $\mathrm{Gr}=\mathrm{Gr}_{0}$, the Marangoni num- 72 ber variation range is widened with the increase of $\alpha_{x} .73$ When wave number changes from 3 to 6 , the Marangoni 74 number variation range will enlarge from $[3.11 ; 7.12]_{75}$ to $[-3.45 ; 12.8]$ (see Fig. $7(\mathrm{~b})$, domain $\mathrm{U}_{2}$ ). With in- 76 creasing $\alpha_{x}$ other type of instability will appear. In the 77 system with $h_{1}=4 \mathrm{~mm}$ patterns $I I I$ will be formed 78 at $\mathrm{Gr}=\mathrm{Gr}_{0} \cdot 10^{-1}$ and $\mathrm{Gr}=\mathrm{Gr}_{0}$, upon that in mi- 79 crogravity conditions $\mathrm{Ma} \in[0.94 ; 6.43]$ under $\alpha_{x}=5$, во Ma $\in[-1.19 ; 8.0]$ under $\alpha_{x}=6$. In normal gravity these 81 structure will be observed, if $\alpha_{x}$ is changed from 1 to 82 3 and corresponding ranges of Ma are $[1.03 ; 1.4]$ and $_{83}$ [-1.05; 0] (see Fig. $7(\mathrm{~d})$, domain $\mathrm{U}_{1}$ ). At larger $\alpha_{x}$ other ${ }_{84}$ type of instability appears.

Deformed structures $I V$ appear in the system with 86 the cold thermocline within the liquid layer. Upon that, 87 the basic flow is the mixed type flow (the reverse mo- 88 tion is observed near the interface). The patterns are 89 characterized by the marked chessboard pattern of the 90 thermal cells in the liquid layer. The upper rows of the 91 thermal spots are similar to patterns $I$ and the lower 92 line of the cells is formed due to the convective mech- 93 anism, which is a dominant one. Patterns $I V$ appear 94 only in the terrestrial conditions or under hypergravity 95 in the systems with $h_{1}=4$ or $h_{1}=3 \mathrm{~mm}$, respectively. ${ }_{96}$ In the first case $\mathrm{Gr}=\mathrm{Gr}_{0}$, wave number is changed ${ }_{97}$ from 1 to 5 and appropriate ranges of the Marangoni ${ }_{98}$ number variations are $[0 ; 24.76]$ and $[0 ; 36.66]$. In the ${ }_{99}$ system with $h_{1}=3 \mathrm{~mm}$ this instability type occurs at $\mathrm{t}_{100}$ $\mathrm{Gr}=2 \mathrm{Gr}_{0}$, upon that Ma $\in[0 ; 43.01]$ under $\alpha_{x}=2{ }_{101}$ $\mathrm{Ma} \in[0 ; 51.62]$ under $\alpha_{x}=3$.

102

Mixed type structures $V$ are formed in the systemsios with the thermocline within the liquid and with quite a104 large liquid layer thickness. Here, the cold thermoclineros is located near the interface and the lower unsteady $y_{106}$ stratified zone occupies a large part of the liquid layer. The basic mechanism of the formation of patterns $V$ is the convective one. In the system with $h_{1}=5 \mathrm{~mm}$ the instability mode will be realized at $\mathrm{Gr}=\mathrm{Gr}_{0} \cdot 10^{-1}$, if Ma $<7.37$ under $\alpha_{x}=3$ and Ma $<8.14$ under $\alpha_{x}=4$. At larger $\alpha_{x}$ other type of instability appears.

Deformed near-surface vortex structures $V I$ arise in the systems with quite a large liquid layer thickness and hot thermocline within the liquid layer. This temperature distribution is formed under a considerable thermal load; here, the hot thermocline is very close to the interface. Thus, the thin near-surface layer with the unstable temperature stratification emerges and the convective motion occurs in this stripe. Due to the significant positive thermal load the intensive reverse flow occurs in the liquid layer. Patterns VI are deformed due to the interaction with the basic flow. The instability mode arises in the systems with $h_{1}=5$ and $h_{1}=6 \mathrm{~mm}$ only at $\mathrm{Gr}=\mathrm{Gr}_{0} \cdot 10^{-1}$. For the first configuration the structures are formed under $\alpha_{x}=15$ and $\mathrm{Ma}=134.81$. If $h_{1}=6 \mathrm{~mm}$ we can observe these patterns under $\alpha_{x}=11$ and $\mathrm{Ma}=122.21$. We emphasize that at larger $\mathrm{Ma}$ for each liquid layer thickness the exact solution predicts infeasible values of the vapor concentration function. Thus, instability domains $\mathrm{U}_{2}$ and $\mathrm{U}_{3}$ situated above curves 2 and 3 in Fig. 7, where this instability mode can be realized, just are theoretical "solutions" of the problem stability under study.

Twin vortex structures VII are originated by the convective mechanism in the systems with the unsteady stratified liquid. The distribution of this type are observed only under hypergravity or in the terrestrial conditions and only for the flows of the Poiseuille's type. The thermal cells are deformed by the basic flow, they are elongated in the direction of the main motion. Therefore, two rows of the vortex structures appear due to the interaction distributions with the basic flow. The upper and lower vortices have the opposite circulation. The instability mode appears in the system with $h_{1}=5$ at $\mathrm{Gr}=\mathrm{Gr}_{0}$ and $\mathrm{Ma}>0$ in the whole range of wave numbers under study.

Typical convective cells VIII are formed in the unsteady stratified liquid. The form of disturbances is observed in the system with $h_{1}=6 \mathrm{~mm}$, when the thermocapillary effect is entirely suppressed by gravity and the interface temperature is cooled due to evaporation. The basic driving mechanism is the convective one for patterns VIII to appear. The structures are formed under the weak positive thermal load, i. e. at quite small positive longitudinal temperature gradients $A$. Only long wave perturbations with $\alpha_{x} \leq 1.1$ lead to the appearance of the structure under $\mathrm{Ma} \leq 6.92$. 
Table 3 Typical forms of the most dangerous perturbations 41

\begin{tabular}{lllll}
\hline \multirow{2}{*}{$h_{1}, \mathrm{~mm}$} & $\mathrm{U}$ & $g_{0} \cdot 10^{-1}$ & $g_{0}$ & $2 g_{0}$ \\
\hline \multirow{2}{*}{2} & $\mathrm{U}^{+}$ & $I$ & $I I,\left(^{*}\right)$ & $I I,\left(^{*}\right)$ \\
\cline { 2 - 5 } & $\mathrm{U}^{-}$ & $I$ & $I I,\left(^{*}\right)$ & $I I,\left(^{*}\right)$ \\
\hline \multirow{2}{*}{3} & $\mathrm{U}^{+}$ & $I I,\left(^{*}\right)$ & $I I I,\left(^{*}\right)$ & $I V$ \\
\cline { 2 - 5 } & $\mathrm{U}^{-}$ & $I I,\left(^{*}\right)$ & $I I I,\left(^{*}\right)$ & $I I,\left(^{*}\right)$ \\
\hline \multirow{2}{*}{4} & $\mathrm{U}^{+}$ & $I I I,\left(^{*}\right)$ & $I V$ & - \\
\cline { 2 - 5 } & $\mathrm{U}^{-}$ & $I I I,\left(^{*}\right)$ & $I I I,\left(^{*}\right)$ & - \\
\hline \multirow{2}{*}{5} & $\mathrm{U}^{+}$ & $V I$ & $V I I$ & - \\
\cline { 2 - 5 } & $\mathrm{U}^{-}$ & $V,\left(^{*}\right)$ & $I$ & - \\
\hline \multirow{2}{*}{6} & $\mathrm{U}^{+}$ & $V I$ & $I$ & - \\
\cline { 2 - 5 } & $\mathrm{U}^{-}$ & $V I I I,\left(^{* *}\right),\left(^{*}\right)$ & $I$ & - \\
\hline
\end{tabular}

We specify mixed type patterns $I X$, which are slightly ${ }^{53}$ similar in structure to patterns $I V$. Perturbations $I X^{54}$ are formed under the following conditions: the thick- ${ }^{55}$ ness of the liquid layer is $h_{1}=6 \mathrm{~mm}$, cold thermocline ${ }^{56}$ is in the liquid layer, system is in the weak gravitational ${ }^{57}$ field. They are observed in the narrow range of the wave ${ }^{58}$ number $2 \leq \alpha_{x} \leq 4$ and of thermal loads. The values of ${ }^{59}$ the longitudinal temperature gradients $A$ are such that ${ }^{60}$ the weak reverse motion arises in the thin near-surface ${ }^{61}$ zone due to the Marangoni effect, which, however, re- ${ }^{62}$ mains weak at the liquid layer thickness under consider- ${ }^{63}$ ation. Structures $I X$ are characterized by the presence ${ }^{64}$ of the clearly defined double thermal spots with the ${ }^{65}$ cores both on the interface and within the liquid layer. ${ }^{66}$ The formation of the double thermal structures is $\mathrm{ex}^{-67}$ plained by the coexistence of thermocapillary effect and ${ }^{68}$ unstable temperature stratification in the lower part of ${ }^{69}$ the liquid layer. The Marangoni effect is weak to form the essential reverse flow, but sufficient for the appearance of the thermocapillary structures at the interface. ${ }^{70}$ The presence of the cold thermocline in the lower fluid results in the formation of the thermal patches within ${ }^{7}$ the liquid. For the system under study the instability mode is realized at $\mathrm{Gr}=\mathrm{Gr}_{0} \cdot 10^{-1}$ under $\alpha_{x}=2$ with $\mathrm{Ma}<9.17$, under $\alpha_{x}=3$ with $\mathrm{Ma}<9.62, \alpha_{x}=4$ with $\mathrm{Ma}<9.77$ (see domain $\mathrm{U}_{3}$ below curve 3 in the specified range of values $\alpha_{x}$ in Fig. 7).

The analysis results for the typical forms of the appearing instability are presented in Table 3, where the type of the arising perturbations and ascertained regularities of the subsequent transition from one type to another with the changing wave number $\alpha_{x}$ are specified for all the considered values of the gravity acceleration and liquid layer thickness. The symbols $(*)$ and $(* *)$ indicate that the transition to structures $I$ and $I X$, respectively, occurs with the increase of the wave number $\alpha_{x}$. For $h_{1}=4,5,6 \mathrm{~mm}$ the hypergravity conditions were not considered due to the above mentioned restrictions concerning the conditions of applicability of the solution under study (Sec. 3.2).

\section{Conclusions}

42 The characteristics of the two-layer flows with evaporation at the thermocapillary interface in an infinite channel with the applied boundary thermal load are investigated on the basis of the exact solution in the frame of the Oberbeck-Boussinesq model. In the microgravity condition, the basic factor defining the flow topology and temperature field pattern is the Marangoni effect. It retains the reverse flow and stable temperature stratification in the liquid layer and can lead to the thermocapillary instability. With the increase of the gravity action and thickness layer, the action of the thermocapillary effect abates and the liquid becomes gravitationally unstable and stratified due to evaporation. The coexistence of the thermocapillary and convective mechanisms of instability can be observed in various configurations. Here, different types of the characteristic perturbations can arise in the system. The structures of the thermocapillary, vortex and convective types with different topology and localization of thermal patches are specified, depending on the type of the basic flow, liquid layer thickness and intensity of the gravity action. For all the configurations under study there exists a thermal load in which the basic flow becomes unstable. For most of them the stability loss is accompanied by the formation of the shortwave perturbations of the thermocapillary type. Heat and mass transfer due to evaporation/condensation at the interface has a destabilizing effect.

\section{Appendix}

\subsection{Unknown function form}

Distributions of the velocity, temperature and pressure in the liquid layer are defined by the following formulae:

$$
\begin{gathered}
u_{1}=\frac{y^{4}}{24} \frac{g \beta_{1} a_{2}^{1}}{\nu_{1}}+\frac{y^{3}}{6} \frac{g \beta_{1} A}{\nu_{1}}+\frac{y^{2}}{2} c_{1}+y c_{2}+c_{3}, \\
T_{1}=\left(A+a_{2}^{1} y\right) x+\frac{y^{7}}{1008}\left\{\frac{g \beta_{1}\left(a_{2}^{1}\right)^{2}}{\nu_{1} \chi_{1}}\right\}+\frac{y^{6}}{144}\left\{\frac{g \beta_{1} A a_{2}^{1}}{\nu_{1} \chi_{1}}\right\}+ \\
+\frac{y^{5}}{120} \frac{1}{\chi_{1}}\left\{\frac{g \beta_{1}(A)^{2}}{\nu_{1}}+3 a_{2}^{1} c_{1}\right\}+\frac{y^{4}}{24} \frac{1}{\chi_{1}}\left\{A c_{1}+2 a_{2}^{1} c_{2}\right\}+ \\
+\frac{y^{3}}{6} \frac{1}{\chi_{1}}\left\{A c_{2}+a_{2}^{1} c_{3}\right\}+\frac{y^{2}}{2} \frac{A}{\chi_{1}} c_{3}+y c_{4}+c_{5}, \\
p_{1}^{\prime}=\left(\frac{y^{2}}{2} \rho_{1} g \beta_{1} a_{2}^{1}+y \rho_{1} g \beta_{1} A+\nu_{1} \rho_{1} c_{1}\right) x+\frac{y^{8}}{8} k_{7}+ \\
+\frac{y^{7}}{7} k_{6}+\frac{y^{6}}{6} k_{5}+\frac{y^{5}}{5} k_{4}+\frac{y^{4}}{4} k_{3}+\frac{y^{3}}{3} k_{2}+\frac{y^{2}}{2} k_{1}+y k_{0}+c_{8} .
\end{gathered}
$$


The coefficients, which do not depend on $y$, are the following:

$$
\begin{gathered}
k_{7}=\frac{1}{1008}\left(g \beta_{1} a_{2}^{1}\right)^{2} \frac{\rho_{1}}{\nu_{1} \chi_{1}}, \quad k_{6}=\frac{1}{144}\left(g \beta_{1}\right)^{2} \frac{\rho_{1}}{\nu_{1} \chi_{1}} a_{2}^{1} A, \\
k_{5}=\frac{1}{120} \frac{g \rho_{1} \beta_{1}}{\chi_{1}}\left(\frac{g \beta_{1}(A)^{2}}{\nu_{1}}+3 a_{2}^{1} c_{1}\right), \\
k_{4}=\frac{1}{24} \frac{g \rho_{1} \beta_{1}}{\chi_{1}}\left(A c_{1}+2 a_{2}^{1} c_{2}\right), \\
k_{3}=\frac{1}{6} \frac{g \rho_{1} \beta_{1}}{\chi_{1}}\left(A c_{2}+a_{2}^{1} c_{3}\right), \quad k_{2}=\frac{1}{2} \frac{g \rho_{1} \beta_{1}}{\chi_{1}} A c_{3}, \\
k_{1}=g \rho_{1} \beta_{1} c_{4}, \quad k_{0}=g \rho_{1} \beta_{1} c_{5} .
\end{gathered}
$$

The distributions of the velocity, temperature, pressure and vapor concentration in the upper layer are given by the following expressions:

$$
\begin{aligned}
& u_{2}=\frac{y^{4}}{24} \frac{g}{\nu_{2}}\left(\beta_{2} a_{2}^{2}+\gamma b_{2}\right)+\frac{y^{3}}{6} \frac{g}{\nu_{2}}\left(\beta_{2} A+\gamma b_{1}\right)+\frac{y^{2}}{2} \bar{c}_{1}+y \bar{c}_{2}+\bar{c}_{3}, \\
& T_{2}=\left(A+a_{2}^{2} y\right) x+\frac{y^{7}}{1008} B_{2} \frac{g}{\nu_{2}}\left(\beta_{2} a_{2}^{2}+\gamma b_{2}\right)+ \\
& +\frac{y^{6}}{720}\left[B_{1} \frac{g}{\nu_{2}}\left(\beta_{2} a_{2}^{2}+\gamma b_{2}\right)+4 B_{2} \frac{g}{\nu_{2}}\left(\beta_{2} A+\gamma b_{1}\right)\right]+ \\
& +\frac{y^{5}}{120}\left[B_{1} \frac{g}{\nu_{2}}\left(\beta_{2} A+\gamma b_{1}\right)+3 B_{2} \bar{c}_{1}\right]+\frac{y^{4}}{24}\left[B_{1} \bar{c}_{1}+2 B_{2} \bar{c}_{2}\right]+ \\
& +\frac{y^{3}}{6}\left[B_{1} \bar{c}_{2}+B_{2} \bar{c}_{3}\right]+\frac{y^{2}}{2} B_{1} \bar{c}_{3}+y \bar{c}_{4}+\bar{c}_{5}, \\
& p_{2}^{\prime}=\left[\frac{y^{2}}{2}\left(\rho_{2} g \beta_{2} a_{2}^{2}+\rho_{2} g \gamma b_{2}\right)+y\left(\rho_{2} g \beta_{2} A+\rho_{2} g \gamma b_{1}\right)+\right. \\
& \left.+\rho_{2} \nu_{2} \bar{c}_{1}\right] x+\frac{y^{8}}{8} \bar{k}_{7}+\frac{y^{7}}{7} \bar{k}_{6}+\frac{y^{6}}{6} \bar{k}_{5}+\frac{y^{5}}{5} \bar{k}_{4}+ \\
& +\frac{y^{4}}{4} \bar{k}_{3}+\frac{y^{3}}{3} \bar{k}_{2}+\frac{y^{2}}{2} \bar{k}_{1}+y \bar{k}_{0}+\bar{c}_{8}, \\
& C=\left(b_{1}+b_{2} y\right) x+\frac{y^{7}}{1008} \frac{g}{\nu_{2}}\left(\beta_{2} a_{2}^{2}+\gamma b_{2}\right)\left\{\frac{b_{2}}{D}-\alpha B_{2}\right\}+ \\
& +\frac{y^{6}}{720} \frac{g}{\nu_{2}}\left\{\left[\frac{b_{1}}{D}-\alpha B_{1}\right]\left(\beta_{2} a_{2}^{2}+\gamma b_{2}\right)+4\left[\frac{b_{2}}{D}-\alpha B_{2}\right] \times\right. \\
& \left.\times\left(\beta_{2} A+\gamma b_{1}\right)\right\}+\frac{y^{5}}{120}\left\{\frac{g}{\nu_{2}}\left(\beta_{2} A+\gamma b_{1}\right)\left[\frac{b_{1}}{D}-\alpha B_{1}\right]+\right. \\
& \left.+3\left[\frac{b_{2}}{D}-\alpha B_{2}\right] \bar{c}_{1}\right\}+\frac{y^{4}}{24}\left\{\left[\frac{b_{1}}{D}-\alpha B_{1}\right] \bar{c}_{1}+2\left[\frac{b_{2}}{D}-\alpha B_{2}\right] \bar{c}_{2}\right\}+ \\
& +\frac{y^{3}}{6}\left\{\left[\frac{b_{1}}{D}-\alpha B_{1}\right] \bar{c}_{2}+\left[\frac{b_{2}}{D}-\alpha B_{2}\right] \bar{c}_{3}\right\}+ \\
& +\frac{y^{2}}{2}\left\{\frac{b_{1}}{D}-\alpha B_{1}\right\} \bar{c}_{3}+y \bar{c}_{6}+\bar{c}_{7} .
\end{aligned}
$$

Here

$$
\bar{k}_{7}=\frac{1}{1008} \frac{\rho_{2} g^{2}}{\nu_{2}}\left(\beta_{2} a_{2}^{2}+\gamma b_{2}\right)\left[\left(\beta_{2}-\alpha \gamma\right) B_{2}+\frac{\gamma b_{2}}{D}\right]
$$

$$
\begin{aligned}
\bar{k}_{6}=\frac{1}{720} \frac{\rho_{2} g^{2}}{\nu_{2}}\left\{\left(\beta_{2} a_{2}^{2}+\gamma b_{2}\right)\left[B_{1}\left(\beta_{2}-\alpha \gamma\right)+\frac{\gamma b_{1}}{D}\right]+\right. \\
\left.+4\left(\beta_{2} A+\gamma b_{1}\right)\left[B_{2}\left(\beta_{2}-\alpha \gamma\right)+\frac{\gamma b_{2}}{D}\right]\right\}, \\
\bar{k}_{5}=\frac{\rho_{2} g}{120}\left\{\frac{g}{\nu_{2}}\left(\beta_{2} A+\gamma b_{1}\right)\left[B_{1}\left(\beta_{2}-\alpha \gamma\right)+\frac{\gamma b_{1}}{D}\right]+\right. \\
\left.+3\left[B_{2}\left(\beta_{2}-\alpha \gamma\right)+\frac{\gamma b_{2}}{D}\right] \bar{c}_{1}\right\}, \\
\bar{k}_{4}=\frac{\rho_{2} g}{24}\left\{\left[B_{1}\left(\beta_{2}-\alpha \gamma\right)+\frac{\gamma b_{1}}{D}\right] \bar{c}_{1}+\right. \\
\left.+2\left[B_{2}\left(\beta_{2}-\alpha \gamma\right)+\frac{\gamma b_{2}}{D}\right] \bar{c}_{2}\right\}, \\
\bar{k}_{3}=\frac{\rho_{2} g}{6}\left\{\left[B_{1}\left(\beta_{2}-\alpha \gamma\right)+\frac{\gamma b_{1}}{D}\right] \bar{c}_{2}+\right. \\
\left.+\left[B_{2}\left(\beta_{2}-\alpha \gamma\right)+\frac{\gamma b_{2}}{D}\right] \bar{c}_{3}\right\}, \\
\bar{k}_{2}=\frac{\rho_{2} g}{2}\left[B_{1}\left(\beta_{2}-\alpha \gamma\right)+\frac{\gamma b_{1}}{D}\right] \bar{c}_{3},
\end{aligned}
$$$$
\bar{k}_{1}=\rho_{2} g \beta_{2} \bar{c}_{4}+\rho_{2} g \gamma_{2} \bar{c}_{6}, \quad \bar{k}_{0}=\rho_{2} g \beta_{2} \bar{c}_{5}+\rho_{2} g \gamma \bar{c}_{7} .
$$$$
B_{1}=\frac{D A-\chi_{2} \delta b_{1}}{D \chi_{2}(1-\alpha \delta)}, \quad B_{2}=\frac{D a_{2}^{2}-\chi_{2} \delta b_{2}}{D \chi_{2}(1-\alpha \delta)} .
$$

7.2 Determination of integration constants

$$
b_{2} x+\phi^{\prime}(y)+\alpha a_{2}^{2} x+\alpha \vartheta_{2}^{\prime}(y)=0
$$

and

$$
\left\{\begin{array}{l}
b_{2}+\alpha a_{2}^{2}=0 \\
\phi^{\prime}\left(h_{2}\right)+\alpha \vartheta_{2}^{\prime}\left(h_{2}\right)=0 .
\end{array} \Rightarrow b_{2}=-\alpha a_{2}^{2}\right.
$$

The conditions of velocity and temperature continuity (2.9) result in the relations

$$
c_{3}=\bar{c}_{3}, \quad c_{5}=\bar{c}_{5} .
$$

Due to the linear temperature distribution on the rigid walls (2.7) we have

$$
\vartheta_{1}\left(-h_{1}\right)=\vartheta^{-}, \quad \vartheta_{2}\left(h_{2}\right)=\vartheta^{+}
$$

$a_{2}^{1}=\frac{A-A_{1}}{h_{1}}, \quad a_{2}^{2}=\frac{A_{2}-A}{h_{2}}$,

The mass balance condition leads to the following relations

7. $M=-D \rho_{2}\left(\bar{c}_{6}+\alpha \bar{c}_{4}\right), \quad b_{2}+\alpha a_{2}^{2}=0$.

The following equations are the consequence of the heat transfer condition (2.12) at the interface $y=0$ :

$$
\kappa_{1} a_{2}^{1}-\kappa_{2} a_{2}^{2}-\delta \kappa_{2} b_{2}=0,
$$

$\kappa_{1} c_{4}-\kappa_{2} \bar{c}_{4}-\delta \kappa_{2} \bar{c}_{6}=-\lambda M$, 
The first equality defines the following relation between $a_{2}^{1}$ and $a_{2}^{2}$ :

$$
a_{2}^{2}=K_{a} a_{2}^{1}, \quad K_{a}=\frac{\kappa_{1}}{\kappa_{2}(1-\alpha \delta)} .
$$

1 Herein condition (A.1) is taken into account. Since $a_{2}^{1}$ and $a_{2}^{2}$ are expressed in terms of $A, A_{1}$ and $A_{2}$ (see ${ }^{9}$ (A.2)), then, the following correlation is valid:

$A=\frac{A_{2}+\frac{h_{2}}{h_{1}} K_{a} A_{1}}{1+\frac{h_{2}}{h_{1}} K_{a}}$.

5 The case of the equal longitudinal temperature gradients can be realized $A=A_{1}=A_{2}$, so that $a_{2}^{1}=a_{2}^{2}$.

The consequence of the Clayperon-Clausius equation in the linearized form (2.13) leads to the equalities

$$
b_{1}=C_{*} \varepsilon A, \quad \bar{c}_{7}=C_{*}+C_{*} \varepsilon\left(\bar{c}_{5}-T_{0}\right) .
$$

From dynamic conditions (2.11) it follows that

$$
c_{2}=\frac{\rho_{2} \nu_{2}}{\rho_{1} \nu_{1}} \bar{c}_{2}+\frac{\sigma_{T} A}{\rho_{1} \nu_{1}}, \quad c_{1}=\frac{\rho_{2} \nu_{2}}{\rho_{1} \nu_{1}} \bar{c}_{1} .
$$

The system of equations to determine the unknown integration constants $\bar{c}_{1}, \bar{c}_{2}, \bar{c}_{3}$ results from no-slip conditions (2.6) and conditions of the given gas flow rate (2.14):

$$
\begin{gathered}
\frac{l^{2}}{2} \frac{\rho_{2} \nu_{2}}{\rho_{1} \nu_{1}} \bar{c}_{1}-l \frac{\rho_{2} \nu_{2}}{\rho_{1} \nu_{1}} \bar{c}_{2}+\bar{c}_{3}=l \frac{\sigma_{T} A}{\rho_{1} \nu_{1}}-\frac{g \beta_{1}}{\nu_{1}}\left(\frac{l^{4}}{24} a_{2}^{1}-\frac{l^{3}}{6} A\right), \\
\frac{h^{2}}{2} \bar{c}_{1}+h \bar{c}_{2}+\bar{c}_{3}= \\
-\frac{g}{\nu_{2}}\left(\frac{h^{4}}{24}\left(\beta_{2} a_{2}^{2}+\gamma b_{2}\right)+\frac{h^{3}}{6}\left(\beta_{2} A+\gamma b_{1}\right)\right) \\
\frac{\frac{h^{3}}{6} \bar{c}_{1}+\frac{h^{2}}{2} \bar{c}_{2}+h \bar{c}_{3}=}{\rho_{2}}-\frac{g}{\nu_{2}}\left(\frac{h^{5}}{120}\left(\beta_{2} a_{2}^{2}+\gamma b_{2}\right)+\frac{h^{4}}{24}\left(\beta_{2} A+\gamma b_{1}\right)\right)
\end{gathered}
$$

If $\bar{c}_{1}, \bar{c}_{2}, \bar{c}_{3}$ have been calculated, then $c_{1}, c_{2}, c_{3}$ can be found.

In view of the exact solution form and the second equality in (A.1), we have the relationship between the constants $\bar{c}_{4}$ and $\bar{c}_{6}$ :

$$
\begin{gathered}
\alpha \bar{c}_{4}+\bar{c}_{6}=F \\
F=-\frac{h^{6}}{144} \frac{g}{\nu_{2}} \frac{b_{2}}{D} a_{2}^{2}\left(\beta_{2}-\alpha \gamma\right)- \\
-\frac{h^{5}}{120} \frac{g}{\nu_{2}}\left(\frac{b_{1}}{D} a_{2}^{2}\left(\beta_{2}-\alpha \gamma\right)+4 \frac{b_{2}}{D}\left(\beta_{2} A+\gamma b_{1}\right)\right)- \\
-\frac{h^{4}}{24}\left(\frac{g}{\nu_{2}} \frac{b_{1}}{D}\left(\beta_{2} A+\gamma b_{1}\right)+3 \frac{b_{2}}{D} \bar{c}_{1}\right)-
\end{gathered}
$$

$$
\begin{gathered}
-\frac{h^{3}}{6}\left(\frac{b_{1}}{D} \bar{c}_{1}+2 \frac{b_{2}}{D} \bar{c}_{2}\right)- \\
-\frac{h^{2}}{2}\left(\frac{b_{1}}{D} \bar{c}_{2}+\frac{b_{2}}{D} \bar{c}_{3}\right)-h \frac{b_{1}}{D} \bar{c}_{3} .
\end{gathered}
$$

The mass of the evaporating liquid is calculated with the help of the first from the sequences of the mass balance equation (A.3): $M=-D \rho_{2} F$.

The second equality from (A.4) sets the dependence of the integration constant $c_{4}$ on $\bar{c}_{4}$ and $\bar{c}_{6}$ :

$$
c_{4}=\frac{\kappa_{2}}{\kappa_{1}} \bar{c}_{4}+\frac{\delta \kappa_{2}}{\kappa_{1}} \bar{c}_{6}-\frac{\lambda M}{\kappa_{1}} .
$$

The constants $\bar{c}_{4}, \bar{c}_{6}$ and $c_{5}$ are determined from the system of equations being the result of the conditions for the temperature on the solid channel walls $\vartheta_{1}\left(-h_{1}\right)=$ $\vartheta^{-}, \vartheta_{2}\left(h_{2}\right)=\vartheta^{+}($see $(\mathrm{A} .2))$ and mass balance equation (A.3):

$$
\begin{aligned}
& -l \frac{\kappa_{2}}{\kappa_{1}} \bar{c}_{4}-l \frac{\delta \kappa_{2}}{\kappa_{1}} \bar{c}_{6}+c_{5}=\vartheta^{-}+\frac{l^{7}}{1008} \frac{g \beta_{1}\left(a_{2}^{1}\right)^{2}}{\nu_{1} \chi_{1}}- \\
& -\frac{l^{6}}{144} \frac{g \beta_{1} A a_{2}^{1}}{\nu_{1} \chi_{1}}+\frac{l^{5}}{120}\left(\frac{g \beta_{1} A^{2}}{\chi_{1} \nu_{1}}+\frac{3 a_{2}^{1}}{\chi_{1}} c_{1}\right)- \\
& \frac{l^{4}}{24}\left(\frac{A}{\chi_{1}} c_{1}+\frac{2 a_{2}^{1}}{\chi_{1}} c_{2}\right)+\frac{l^{3}}{6}\left(\frac{A}{\chi_{1}} c_{2}+\frac{a_{2}^{1}}{\chi_{1}} c_{3}\right)- \\
& -\frac{l^{2}}{2} \frac{A}{\chi_{1}} c_{3}-l \frac{\lambda M}{\kappa_{1}} \\
& h \bar{c}_{4}+c_{5}=\vartheta^{+}-\frac{h_{7}}{1008} B_{2} \frac{g}{\nu_{2}}\left(\beta_{2} a_{2}^{2}+\gamma b_{2}\right)- \\
& -\frac{h^{6}}{720} \frac{g}{\nu_{2}}\left[B_{1}\left(\beta_{2} a_{2}^{2}+\gamma b_{2}\right)++4 B_{2}\left(\beta_{2} A+\gamma b_{1}\right)\right]- \\
& -\frac{h^{5}}{120}\left[B_{1} \frac{g}{\nu_{2}}\left(\beta_{2} A+\gamma b_{1}\right)+3 B_{2} \bar{c}_{1}\right]-\frac{h^{4}}{24}\left[B_{1} \bar{c}_{1}+2 B_{2} \bar{c}_{2}\right]- \\
& -\frac{h^{3}}{6}\left[B_{1} \bar{c}_{2}+B_{2} \bar{c}_{3}\right]-\frac{h^{2}}{2} B_{1} \bar{c}_{3}, \\
& \alpha \bar{c}_{4}+\bar{c}_{6}=F
\end{aligned}
$$

Here, the condition $c_{5}=\bar{c}_{5}$ and the second relationship from (A.3) are taken into account.

A special case $b_{2}=0\left(a_{2}^{2}=a_{2}^{1}=0\right)$ can be realized.

Conflict of Interest: The authors declare that they have no conflict of interest. 


\section{References}

1. Andreev V.K., Kaptsov O.V., Pukhnachov V.V., Rodi- 68 onov A.A., Applications of group theoretical methods in 69 hydrodynamics, 408. Kluwer Academic Publ., Dordrecht, 70 Boston, London (1998)

2. Andreev V.K., Bublik V.V., Bytev V.O., Symmetries ${ }^{72}$ of Nonclassical Models of Hydrodynamics, 352. Nauka, ${ }^{73}$ Novosibirsk (2003) [in Russian]

3. Andreev V.K., Gaponenko Yu.A., Goncharova O.N., ${ }^{75}$ Pukhnachov V.V., Mathematical models of convection ${ }^{76}$ (de Gruyter Studies in Mathematical Physics), 417. De ${ }^{77}$ Gruyter, Berlin/Boston (2012)

4. Bar-Cohen A., Wang P. Thermal managment of on-chip ${ }^{79}$ hot spot, J. Heat Transfer, 134(5), 051017 (2012)

5. Bekezhanova V.B. Convective instability of Marangoni- ${ }^{81}$ Poiseuille flow under a longitudinal temperature gradient, ${ }_{83}^{82}$ J. Appl. Mech. Tech. Phys., 52(1), 74-81 (2011)

6. Bekezhanova V.B. Three-dimensional disturbances of $a_{85}^{84}$ plane-parallel two-layer flow of a viscous, heat-conducting ${ }_{86}^{85}$ fluid, Fluid Dyn., 47(6), 702-708 (2012)

7. Bekezhanova V.B., Goncharova O.N., Stability of the ex- ${ }_{88}^{87}$ act solutions describing the two-layer flows with evapora- ${ }_{89}$ tion at interface, Fluid Dynamics Research, 48(6), $061408_{90}$ (2016)

8. Bekezhanova V.B., Goncharova O.N., Rezanova E.V., She- ${ }_{92}{ }_{91}$ fer I.A., Stability of two-layer fluid flows with evaporation ${ }_{93}$ at the interface, Fluid Dynamics, 52(2), 189-200 (2017)

9. Berg J.C., Acrivos A., Boudart M., Evaporative Convec- 95 tion, Adv Chem Eng., 6, 61-123 (1966)

10. Birikh R.V., Thermocapillary convection in a horizontal 97 layer of liquid, J. Appl. Mech. Tech. Phys., 3, 43-45 (1966) 98

11. Burelbach J.P., Banko S.G., Davis S.H. Nonlinear sta- 99 bility of evaporating/condensing films, J. Fluid Mech, 195,100 463-494 (1988)

12. Colinet P., Joannes L., Iorio C.S., Haute B., Bestehorn102 M., Lebon G., Legros J.-C., Interfacial turbulence in evapo-103 rating liquids: Theory and preliminary results of the ITEL-104 master 9 sounding rocket experiment, Advances in Space105 Research, 32(2), 119-127 (2003)

13. Colinet P., Legros J.C., Velarde M.G., Nonlinear Dy-107 namics of Surface-Tension-Driven Instabilities, 512. Wiley_108 VCH, Berlin (2001)

14. Das K.S., Ward C.A., Surface thermal capacity and its ${ }^{110}$ effects on the boundary conditions at fluid-fluid interfaces, ${ }_{111}^{11}$ Phys. Rev., E 75, 1-4 (2007)

15. Frezzotti A., Boundary conditions at the vapor-liquid $d_{114}^{113}$ interface, Phys. Fluids, 23, 030609 (2011)

16. Godunov S.K., On the numerical solution of boundary ${ }^{115}$ value problems for systems of ordinary linear equations ${ }_{117}$ Uspekhi Matem. Nauk, 16(3(99)), 171-174 (1961)

17. Goncharova O.N. Modeling of Flows Under Conditions of Heat and Mass Transfer at the Interface, Izvestiya of Altai ${ }_{120}^{119}$ State University Journal, 73(1/2), 12-18 (2012)

18. Goncharova O.N., Hennenberg M., Rezanova E.V. ${ }^{121}$ Kabov O.A., Modeling of the convective fluid flows with ${ }_{123}$ evaporation in the two-layer systems, Interfacial Phenomena and Heat Transfer, 1(4), 317-338 (2013)

19. Goncharova O.N., Kabov O.A., Investigation of the two- ${ }_{-126}$ layer fluid flows with evaporation at interface on the basis ${ }_{127}$ of the exact solutions of the 3D problems of convection,128 Journal of Physics: Conference Series, 754, 032008 (2016) 129

20. Goncharova O.N., Rezanova E.V., Example of an exact $t_{130}$ solution of the stationary problem of two-layer flows with 131 evaporation at the interface, J. Appl. Mech. Techn. Phys.,132 55(2), 247-257 (2014)
21. Goncharova O.N., Rezanova E.V., Construction of a Mathematical Model of Flows in a Thin Liquid Layer on the Basis of the Classical Convection Equations and Generalized Conditions on an Interface, Izvestiya of Altai State University Journal, 85(1/1), 70-74 (2015)

22. Goncharova O.N., Rezanova E.V., Lyulin Yu.V., Kabov O.A., Modeling of two-layer liquid-gas flow with account for evaporation, Thermophysics and Aeromechanics, 22(5), 631-637 (2015)

23. Haut B., Colinet P., Surface-tension-driven instability of a liquid layer evaporating into an inert gas, J. Colloid and Interface Science, 285, 296-305 (2005)

24. Hoke B.C., Chen J.C., Mass Transfer in Evaporating Falling Liquid Film Mixtures, AIChE Journal, 38(5), 781787 (1992)

25. Iorio C.S. Goncharova O.N., Kabov O.A. Study of evaporative convection in an open cavity under shear stress flow, Microgravity Sci. Technol., 21(1), 313-320 (2009)

26. Iorio C.S., Kabov O.A., Legros J.-C., Thermal Patterns in evaporating liquid, Microgravity Sci Technol., XIX (3/4) 27-29 (2007)

27. Kabov O.A., Kuznetsov V.V., Kabova Yu.O., Evaporation, Dynamics and Interface Deformations in Thin Liquid Films Sheared by Gas in a Microchannel (Chapter 2), Encyclopedia of Two-Phase Heat Transfer and Flow II: Special Topics and Applications, Volume 1: Special Topics in Boiling in Microchannels / Micro-Evaporator Cooling Systems (Eds J.R. Thome and J. Kim), 57-108. World Scientific Publishing Company, Singapore, (2015)

28. Kabova Yu., Kuznetsov V.V., Kabov O., GambaryanRoisman T., Stephan P., Evaporation of a thin viscous liquid film sheared by gas in a microchannel, Int. J. Heat and Mass Transfer, 68, 527-541 (2014)

29. Kandlikar S.G., Colin S., Peles Y., Garimella S., Pease R.F., Brandner J.J., Tuckerman D.B., Heat transfer in microchannels -2012 status and research needs, J. Heat Transfer 135(9), 091001 (2013)

30. Kimball J.T., Hermanson J.C., Allen J.S., Experimental investigation of convective structure evolution and heat transfer in quasi-steady evaporating liquid films, Phys. Fluids, 24, 052102 (2012)

31. Klentzman J., Ajaev V.S., The effect of evaporation on fingering instabilities, Phys. Fluids., 21(12), 122101 (2009)

32. Kuznetsov V.V., Heat and Mass Transfer on a LiquidVapor Interface, Fluid Dynamics, 46(5), 754-763 (2011)

33. Kuznetsov V.V., Andreev V.K. Liquid film and gas flow motion in a microchannel with evaporation, Thermophysics and Aeromechanics, 20(1), 17-28 (2013)

34. Landau L.D., Lifshitz E.M., Course of Theoretical Physics, Volume 6: Fluid Mechanics, $2^{\text {nd }}$ Ed., 554. Pergamon Press, Oxford (1987)

35. Li P., Chen Z., Shi J., Numerical Study on the Effects of Gravity and Surface Tension on Condensation Process in Square Minichannel, Microgravity Sci. Technol., 30, 19-24 (2018)

36. Liu R., Kabov O.A., Instabilities in a horizontal liquid layer in co-current gas flow with an evaporating interface, Physical Review E-Statistical, Nonlinear, and Soft Matter Physics, 85(6), 066305 (2012)

37. Lyulin Y., Kabov O., Evaporative convection in a horizontal liquid layer under shear-stress gas flow, Int. J. Heat Mass Transfer, 70, 599-609 (2014)

38. Lyulin Y., Kabov O., Measurement of the evaporation mass flow rate in a horizontal liquid layer partly opened into flowing gas, Tech. Phys. Lett., 39 795-797 (2013)

39. Mancini H., Maza D., Pattern formation without heating in an evaporative convection experiment, Europhys Lett., 66(6), $812-818$ (2004) 
40. Margerit J., Colinet. P., Lebon G., Iorio C.S., Legros J.C., 66 Interfacial nonequilibrium and Benard-Marangoni instabil- 67 ity of a liquid - vapor system, Phys. Rev., E 68, 1-14 (2003) 68

41. Merkt D., Bestehorn M., Benard-Marangoni convection 69 in a strongly evaporating field, Physica D, 185, 196-208 70 (2003)

42. Molenkamp T., Marangoni Convection, Mass Transfer 72 and Microgravity, 240. Ph.D. Dissertation, Rijksuniversiteit 73 Groningen, Groningen (1998)

43. Napolitano L.G., Plane Marangoni-Poiseuille flow two ${ }^{75}$ immiscible fluids, Acta Astronautica, 7, 461-478 (1980) 76

44. Narendranath A.D. Hermanson J.C., Kolkka R.W., ${ }^{77}$ Struthers A.A., Allen J.S., The Effect of Gravity on the ${ }^{78}$ Stability of an Evaporating Liquid Film, Microgravity Sci. ${ }^{79}$ Technol., 26(3), 189-199 (2014)

45. Nie Z.H., Kumacheva E., Patterning Surfaces with Func- ${ }^{81}$ tional Polymers, Nature Materials, 7, 277-290 (2008)

46. Nepomnyashchy A.A., Velarde M.G., Colinet P., In- ${ }^{83}$ terfacial phenomena and convection, 360. Chapman $\&^{84}$ Hall/CRC, Boca Raton (2002)

47. Oron A. Nonlinear dynamics of irradiated thin volatile liquid films, Phys. Fluids, 12(1), 29 (2000)

48. Ostroumov G.A., Free convection under the conditions of an internal problem, 286. Gostekhizdat Press, MoscowLeningrad (1952) [in Russian]

49. Oron A., Davis S.H., Bankoff S.C., Long-scale evolution of thin liquid films, Reviews of Modern Physics, 69(3), 931980 (1997)

50. Ozen O., Narayanan R., The physics of evaporative and convective instabilities in bilayer systems: Linear theory, Phys. Fluids, 16(12) 4644 (2004)

51. Prosperetti A. Boundary conditions at a liquid-vapor interface, Mechanica, 14(1), 34-47 (1979)

52. Pukhnachov V.V., A plane steady-state free boundary problem for the Navier-Stokes equations, J. Appl. Mech. Techn. Phys., 13(3), 340-351 (1972)

53. Pukhnachov V.V., Group-theoretical nature of the Birikh's solution and its generalizations, Book of Proc. Symmetry and differential equations, Krasnoyarsk, 180-183 (2000) [in Russian]

54. Pukhnachov V.V., Symmetries in the Navier-Stokes equations, Uspekhi mechaniki 4(1), 6-76 (2006) [in Russian]

55. Puknachov V.V., Thermocapillary convection under low gravity, Fluid Dynamics Transactions, 14, 140-204 (1989)

56. Reutov V.P., Ezersky A.B., Rybushkina G.V., Chernov V.V., Convective structures in a thin layer of an evaporating liquid under an airflow, J. Appl. Mech. Techn. Phys., 48(4), 469-478 (2007)

57. Rezanova E.V., Shefer I.A. Influence of thermal load on the characteristics of a flow with evaporation, J. Appl. Ind. Math., 11(2), 274-283 (2017)

58. Saenz P.J., Valluri P., Sefiane K., Karapetsas G., Matar O.K., Linear and nonlinear stability of hydrothermal waves in planar liquid layers driven by thermocapillarity, Phys. Fluids, 25(9), 094101 (2013)

59. Saenz P.J., Valluri P., Sefiane K., Karapetsas G., Matar O.K., On phase change in Marangoni-driven flows and its effects on the hydrothermal-wave instabilities, Phys. Fluids, 26(2), 024114 (2014)

60. Scheid B., Margerit J., Iorio C.S., Joannes L, Heraud M., Queeckers P.,Dauby P. C., Colinet P., Onset of Thermal Ripples at the Interface of an Evaporating Liquid under a Flow of Inert Gas, Experiments in Fluids, 52, 1107-1119 (2012)
61. Shi W.-Y., Rong S.-M., Feng L., Marangoni Convection Instabilities Induced by Evaporation of Liquid Layer in an Open Rectangular Pool, Microgravity Sci. Technol., 29, 9196 (2017)

62. Shklyaev O.E., Fried E., Stability of an evaporating thin liquid film, J. Fluid Mech., 584, 157-183 (2007)

63. Shliomis M.I., Yakushin V.I., Convection in a twolayers binary system with an evaporation, Collected papers: Uchenye zapiski Permskogo Gosuniversiteta, seriya Gidrodinamika, 4, 129-140 (1972) [in Russian]

64. Sultan E., Boudaoud A., Amat M.B. Evaporation of a thin film: diffusion of the vapour and Marangoni instabilities, J. Fluid Mech, 543, 183-202 (2005)

65. Voropai P.I., Shlepov A.A. Enhancement of reliability and efficiency of reciprocating compressors, 359. Nedra, Moscow (1980)

66. Zeytounian R.Kh., The Benard-Marangoni thermocapillary-instability problem, Usp. Phys. Nauk, 168(3), 259-286 (1998) 